\title{
Viremia and Interaction of Endothelial Cells, Erythrocytes, Leukocytes and Platelets in Type 2 Diabetes Mellitus and COVID-19: A Closer look at Proposed Mechanisms.
}

\author{
Melvin R. Hayden ${ }^{1,2,3^{*}}$ \\ Departments of Internal Medicine ${ }^{1}$, Endocrinology Diabetes and Metabolism ${ }^{2}$, Diabetes and \\ Cardiovascular Disease Center ${ }^{3}$, University of Missouri-Columbia School of Medicine. \\ * Correspondence: mrh29pete@gmail.com; Tel: 5733463019
}

Melvin R. Hayden, MD

E-mail address:

mrh29pete@gmail.com

Home telephone number:

5733463019

Mailing address:

580 Neongwah Bend Road

Camdenton, Missouri

65020

Melvin R. Hayden ${ }^{1-3}$

\begin{abstract}
Viremia in coronavirus disease 2019 (COVID-19) caused by the severe acute respiratory syndrome coronavirus-2 (SARS-CoV-2) is often only discussed in passing and there are very few references detailing its structural mechanisms. In addition to viremia in the classic closed cardiovascular system, the lymphatic system is discussed in relation to a possible "lympho-viremia". The cells that comprise each of these separate but interacting systems will be examined and include endothelial cells, erythrocytes, leukocytes (monocytes/monocyte-derived macrophages and resident tissue macrophages) (lymphocytes) (neutrophils) and thrombocytes platelets. The SARS-CoV-2 virus has been identified in multiple extrapulmonary target organs at autopsy in those with severe COVID-19 requiring intensive care. Vulnerable COVID-19 patients may suffer from multiple storms including viral/virion storm, redox storm, cytokine storm and thrombo-embolic storm. Therefore, it is important that the possible mechanisms of viremia be explored in greater detail and how these mechanisms might affect intravascular blood components, extracellular tissue interstitium and organ structural remodeling and function. While the co-morbidity of T2DM does not increase the risk of acquiring COVID-19, it is commonly accepted that T2DM increases the risk for COVID-19 admissions to hospitals, assisted ventilation, morbidity and mortality. Importantly, the co-existence of T2DM and COVID-19 may have synergistic detrimental outcomes. WC 199
\end{abstract}

Keywords: COVID-19; Endothelial activation; Inflammation; Leukocytes; Lymphatic system; Lymphocytes; Neutrophil NETs; NETosis; Platelets; RBCs; SARS-CoV-2; T2DM; Thrombosis; Viremia. 


\section{Introduction}

We are now nine months into this novel coronavirus disease $20 \underline{19}$ (COVID-19) pandemic (23 December, 2020) caused by the severe acute $\underline{\mathbf{r}}$ espiratory syndrome coronavirus- $\underline{\mathbf{2}}$ (SARS-CoV2) virus. Therefore, it seems appropriate to place this pandemic in historical perspective. There were two initial and seminal papers from China that introduced the world to this forthcoming pandemic issued by the World Health Organization (WHO) on the $11^{\text {th }}$ of March 2020 [1,2]. In the United States we are currently experiencing exponential increases in newly diagnosed cases (in excess of 18.4 million) and have one of the highest mortality figures (326,150 total deaths) globally according to a New York Times database [3]. Globally, the current total number of cases are in excess of 37,109,851 and the number of deaths may exceed 1,070,355 according to the WHO [4]. This pandemic far exceeds the H1N1 pdm09 virus of 2009 (12,469 deaths) [5] and recent coronavirus epidemic of SARS- CoV 2002-3 (774 deaths), MERS-2012 (886 deaths) as well as the annual influenza 2019-2020 season ( 20,000 deaths) [6]. More and more, the COVID-19 pandemic appears to be paralleling the great pandemic of the 1918 Spanish flu (H1N1 virus) pandemic (an estimated 50 million deaths worldwide and $\sim 675,000$ deaths in the United States) according to the Center of Disease Control (CDC) [7].

Throughout this review, the use of various animal models and their ultrastructural images as well as illustrations will be utilized to assist in understanding the discussion of both the vascular and lymphatic systems origin of viremia, since human tissues are not readily available to study.

The pulmonary blood-air and blood-gas barrier (BGB) (Fig. 1) is responsible for the separation of blood components (circulating proteins, erythrocytes, platelets and leukocytes) from the terminal alveolar air sacs and their separating thinned $(200-300 \mathrm{~nm})$ fused basement membranes between alveolar epithelia I or pneumocyte $1(\mathrm{Pn} 1)$ and capillary endothelial cells in order to allow for the rapid exchange of gases (oxygen and carbon dioxide) $[6,8,9,10]$.

The beta coronavirus SARS-CoV-2 (2019-nCoV) infective virus of COVID-19 is known to invade the host via small aerosolized droplets (less than 5 micrometers) and via surface contact. This human-to-human viral spread enters the host primarily through naso-oropharynx and proceeds via the trachea respiratory epithelial cells (ciliated and non-ciliated) with progression to lower airways to primarily infect and damage the lining alveolar epithelia II (AEII) pneumocyte $2(\mathrm{Pn} 2)$. Thus, this progression interferes with the critical exchange of gases (oxygen uptake and carbon dioxide disposal) via the BGB and pulmonary blood flow (PBF). The subsequent remodeling of the BGB allows for the SARS-CoV-2 daughter virions and their viral RNA to enter to blood compartment where it is exposed to multiple cells and proceeds to the systemic circulation allowing for viremia or the detection of SARS-CoV-2 RNA (RNAemia) (Figs. 1, 2) [6, 3, 11].

This critical BGB or alveolar vascular unit (AVU) in the pulmonary tissues is responsible for the ultrastructural capillary PBF to the lung [6]. Oxygen delivery to tissues and removal of metabolic byproducts including carbon dioxide is critical because the entire body including the lung is dependent on a viable oxygen delivery system in order to prevent damaging cellular hypoxia (Figs. 1, 2). 


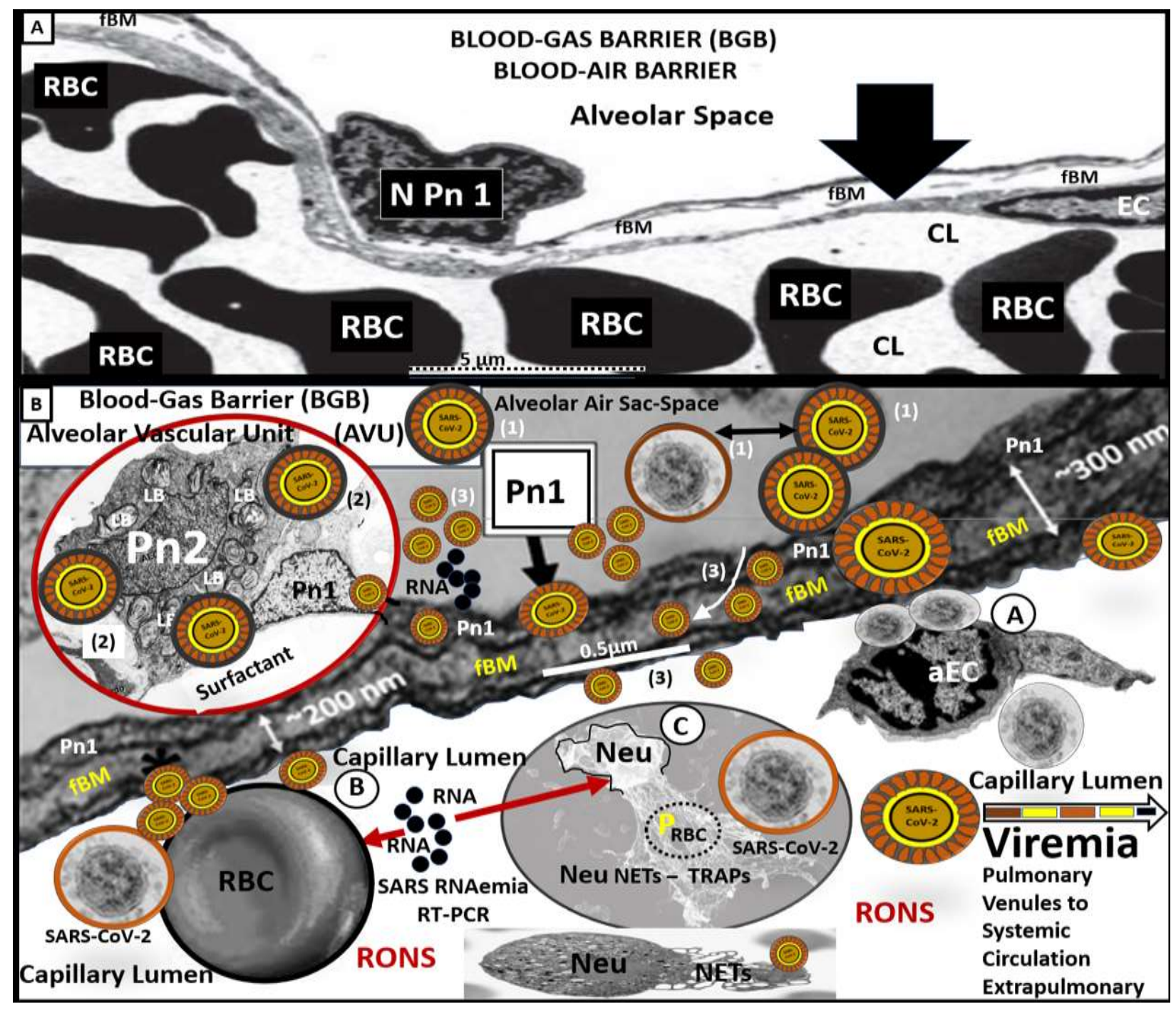

Figure 1. Possible origin of viremia at the pulmonary blood-gas barrier (BGB) as illustrated in vertebrate animal models. Panel A allows for a better understanding with lower ultrastructural magnification of the BGB in relation to the flowing pulmonary blood flow of red blood cell(s) (RBCs) within the lumen of the capillary of the developing marsupial quokka wallaby lung [9]. Panel B allows for a higher magnified illustration of the BGB from a nine-week-old Sprague-Dawley rat model with ultrastructure images of numerous intravascular cells [6]. Modified with permission by CC 4.0, respectively [9,6]. Upper panel A illustrates the pneumocyte 1 (Pn1) - alveolar type I (AT-1) squamous epithelial cell of the BGB and its fused basement membrane (fBM) of the endothelial cell (EC) and note its extreme thinness of 200-300 nanometers $(\mathrm{nm})$. Scale bar $=5 \mu \mathrm{m}$. Panel B illustrates the BGB at higher magnification with scale bar of $0.5 \mu \mathrm{m}$ with insertion of multiple cells and icons (not to scale) to illustrate the process of viremia [stages (1)-(3)]. The lung is the primary organ initially infected with the severe acute respiratory syndrome coronavirus-2 (SARS-CoV-2) virus at the terminal bronchioles and alveolar blood-gas barrier or blood-air barrier (BGB) - stage (1) including the alveolar capillary vascular unit (AVU). SARS-CoV-2 primarily infects the larger more cuboidal shaped alveolar epithelial II or pneumocyte 2 (Pn2) - stage 2. Importantly, Pn2 are responsible for the synthesis and production of surfactant and also serve as the progenitor cells to renew and repopulate the damaged Pn1 of the BGB. Herein, the alveolar Pn1 line the alveolar air sacs - spaces and their basement membranes fuse with the basement membranes of 
endothelial cell(s) (EC) to create a very thin fused BM (fBM) creating a 200-300 nm barrier between the alveolar air sacs and the capillary lumen for rapid gas (oxygen and carbon dioxide) exchange at the BGB. The SARS-CoV-2 virus (stage 1) enters the Pn2 and undergoes replication with Pn2 necrosis/apoptosis (stage 2) and are expelled into the alveolar air sacs where they then are capable of invading and or traversing the Pn1 and traverse the fBM to either enter and traverse the ECs via absorptive transcytosis or pass between adjacent ECs via the paracellular route to enter the BGB capillary lumen (stage 3) wherein the $\mathrm{CoV}-2$ virus begins it systemic journey via the pulmonary capillaries and venules to pulmonary veins to the left atrium and ventricle to enter the aorta and disseminate throughout the systemic circulation. It is important to note that once SARS- CoV-2 virus or its viral RNA protein particles (SARS RNAemia) enters the capillary lumen they will be able to immediately interact with capillary ECs (A), RBCs and platelets (not shown) (B) and leukocytes (both the granulated neutrophils Net forming (C) and the nongranulated lymphocytes (not shown). $B G B=$ blood-gas barrier; $C L=$ capillary lumen; $E C=$ endothelial cell(s); $f B M=$ fused basement membranes; $L B=$ lamellar bodies; $N=$ nucleus; $N E T=$ neutrophil extracellular trap(s); NEU = neutrophil; Pn1= pneumocyte 1; Pn2 = pneumocyte 2; $R B C=$ red blood cell(s); RONS = reactive oxygen nitrogen species; SARS-CoV-2 = severe acute respiratory syndrome coronavirus-2.

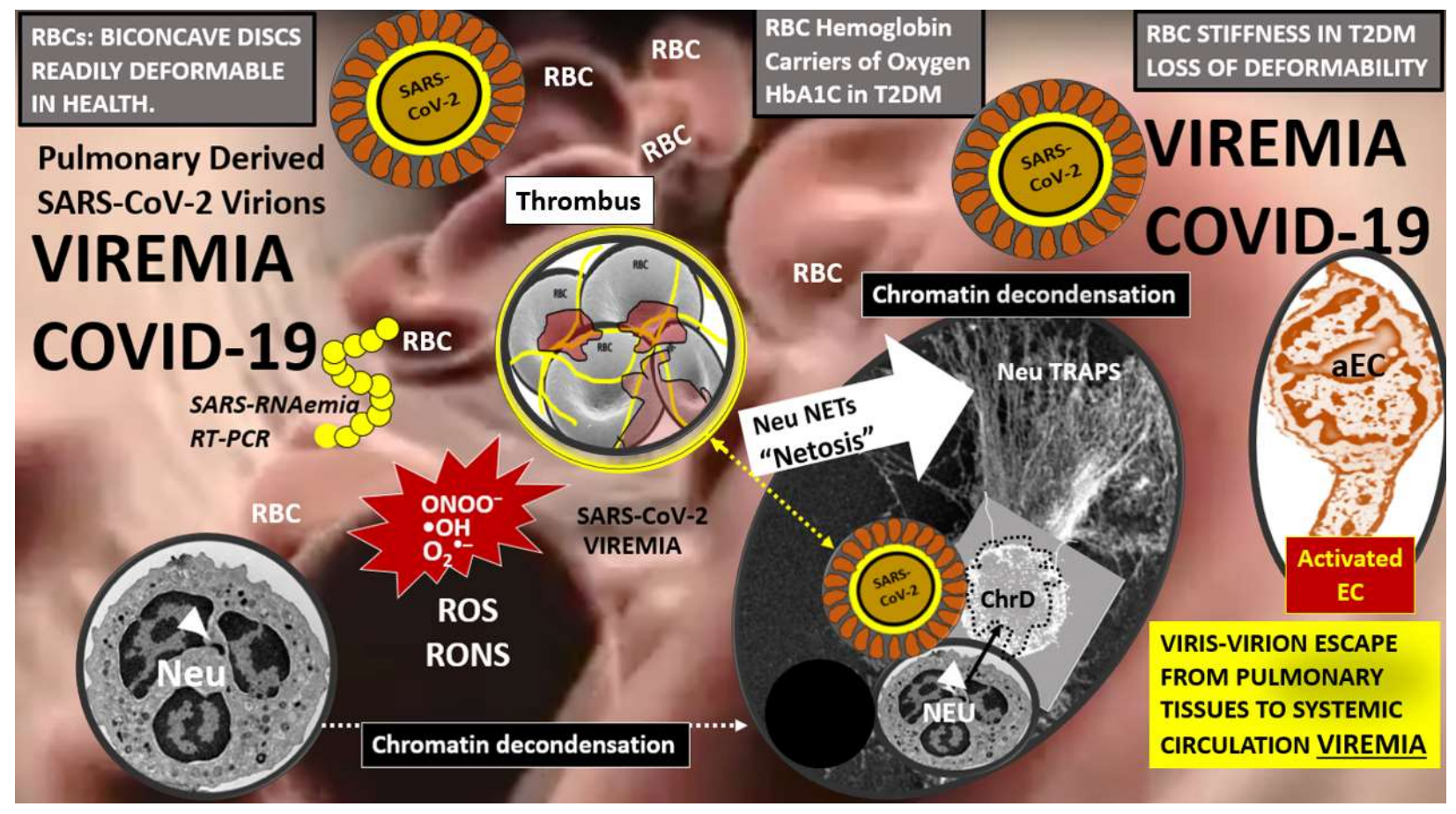

Figure 2. Effect of SARS-CoV-2 viremia on vascular cells. Once the SARS-CoV-2 virus has entered the capillary blood-gas barrier (BGB) lumen, it is capable of having immediate detrimental localized effects on the intravascular endothelial cell(s) (ECs), red blood cell(s) (RBCs), thrombus (platelet - fibrin RBCs) and leukocytes including the formation of neutrophil - neutrophil extracellular traps (NETs). Viremia includes not only the intact SARS-CoV-2 virus but also viral nuclear ribonucleic acid (RNA) and RNAemia as measured by RT-qPCR to evaluate viremic load. Monocytes and lymphocytes were not illustrated but are also involved. $B G B=$ blood-gas barrier; $C h r D=$ chromatin decondensation; $a E C=$ activated endothelial cell(s); RBC = red blood cell(s); NET = neutrophil extracellular trap(s); NEU = neutrophil; RONS = reactive oxygen nitrogen species (superoxide, hydroxyl radical, peroxinitrite and others); $R O S=$ reactive oxygen species; $R T-q P C R=$ quantitative reverse transcription polymerase chain reaction; SARS-CoV-2 = severe acute respiratory syndrome coronavirus-2; SARS-RNAemia = severe acute respiratory syndrome- ribonucleic acidemia. 
It is therefore critical to provide for a more in-depth discussion of viremia and its possible mechanisms occurring in the primary infected pulmonary tissue at the AVU and BGB. Not only because this is the route of viral transmission to the extrapulmonary target organs of COVID-19 (Fig. 3) but also how the intravascular SARS-CoV-2 virus may have immediate direct detrimental effects on the lining endothelial cell(s) (EC) and intravascular blood components such as the erythrocytes (RBCs), leukocytes and platelets. Each of these vital components play a critical role in blood rheology and intravascular coagulation systems that may result in coagulopathies when exposed to SARS-CoV-2 virus and/or its viral RNA.

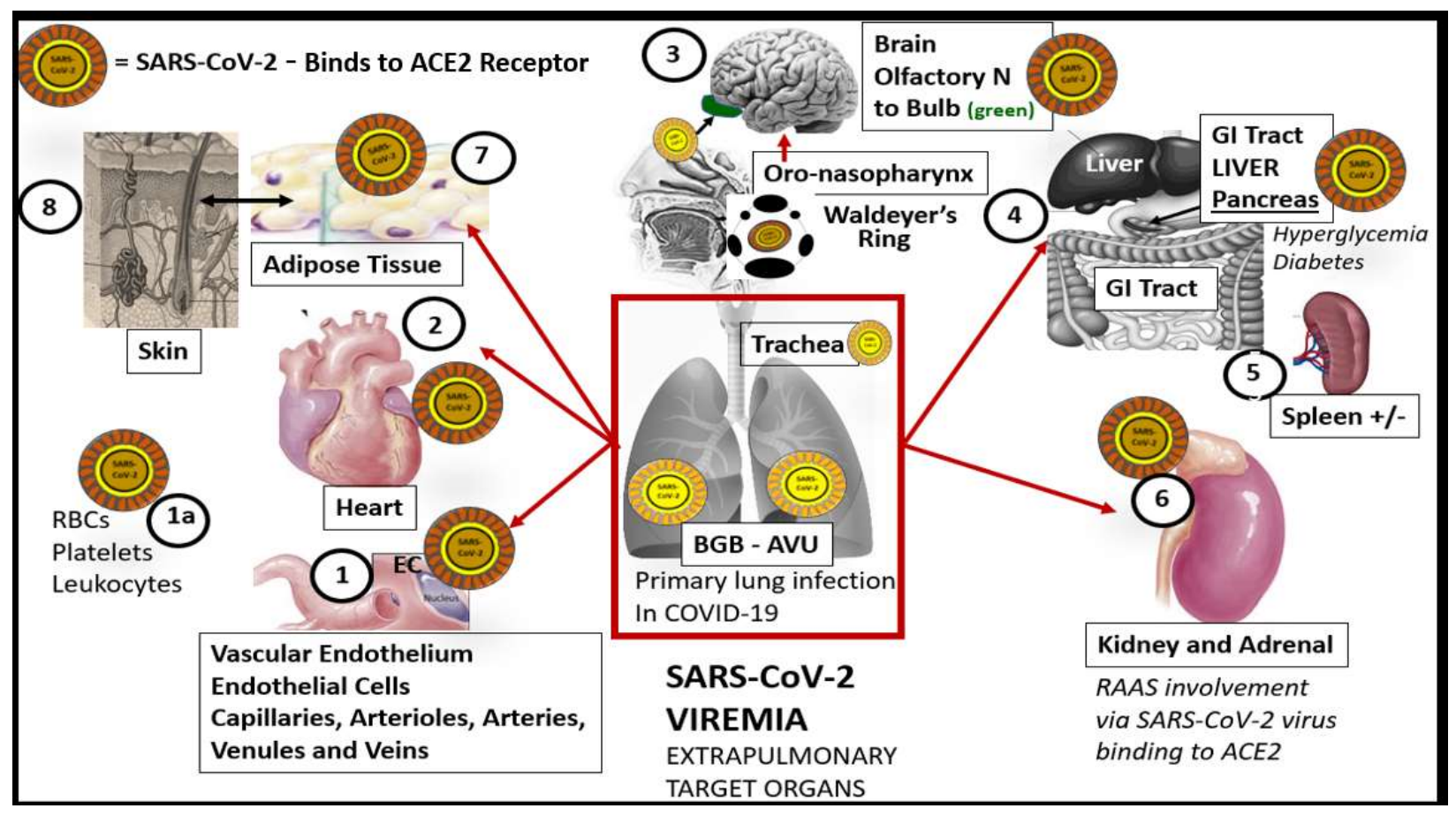

Figure 3. Extrapulmonary target organs affected by SARS-CoV-2 viremia. The angiotensin converting enzyme 2 (ACE2) is known to be the receptor for the SARS-CoV-2 virus to enter host epithelial cells and the ACE2 receptor has been found to be present in numerous extrapulmonary tissues and organs [2, 6 , 11]. This illustration depicts the possible multiple extrapulmonary organs affected by the SARS-CoV-2 viremia from the vascular origin associated with viremia (1-8) and the lymphatic system of Waldeyer's ring. $A C E 2=$ angiotensin-converting enzyme 2; $A V U=$ alveolar vascular unit; $B G B=$ blood-gas barrier; $E C=$ endothelial cell; $G I=$ gastrointestinal; $N=$ nerve; $R A A S=$ renin-angiotensin-aldosteronesystem; SARS-CoV-2 = severe acute respiratory syndrome coronavirus-2.

The SARS-CoV-2 virus not only activates antiviral immune responses in the systemic circulation but also in the pulmonary alveoli air sacs - BGB, interstitial septum and alveolar microvasculature - macrovascular arterioles. Additionally, SARS-CoV-2 virus can also cause an uncontrolled dysfunctional inflammatory response characterized by marked pro-inflammatory cytokine release or cytokine storm in those with severe COVID-19 infections. These factors are important to the central findings of lymphopenia, lymphocyte dysfunction, and granulocyte neutrophil excess and monocyte/monocyte-derived macrophage abnormalities. Importantly, SARS-CoV-2 induced immune abnormalities may lead to septic shock, severe multiple targetorgan dysfunction via viremia and result in dysfunction and damage. SARS-CoV-2 may also 
increase the risk for infections by other microorganisms such as pulmonary bacteria and influenza [12]. The epithelium of the lungs is the largest internal surface in the human body that is in continuous contact with our environment making the pulmonary epithelium especially vulnerable to any novel respiratory virus due to droplets and aerosol inhalation. Of major concern, at this point in time, is the fact that many northern hemisphere countries are now entering the seasonal winter increase in influenza and the possibility that these two respiratory viruses may interact and overwhelm our hospitals and care facilities. While the cells of the human body have developed an extensive array of mechanisms to contain viral infections, certain viruses may develop equal mechanisms to evade their host cellular defenses [13]. Thus, the novel SARS-CoV-2 virus has evolved to become one of the deadliest pandemic viruses known since the great pandemic of the Spanish flu in 1918.

\section{Viremia: The Vascular System}

The efficacy of viral/virion entry into host cells may depend on three crucial points: (i) the degree of contagiousness or basic reproduction number - $\mathrm{R}_{0}$ (a mathematical term that indicates how contagious an infectious disease is) and viral load exposure time of the virus to host, (ii) viral receptors (ACE2 in COVID-19) on host cell membrane and (iii) functionality of immune conditions of the host. Incidentally, the estimated $\mathrm{R}_{0}$ for $\mathrm{H} 1 \mathrm{~N} 1$ of the 1918 Spanish flu was 1.2 2.8, annual seasonal influenza 0.9 - 2.1, swine flu H1N1 of 2009 1.4 -1.6 [14] and the COVID19 pandemic, which thus far, is estimated to have a mean $\mathrm{R}_{0}$ of 5.6, which strongly suggests a much higher infectivity with the SARS-CoV-2 virus [15].

The SARS-CoV-2 virus is known to preferentially infect the pulmonary tissues in the lower respiratory tracts via the naso-oropharynx route due to person-to-person aerosol inhalation where it is known to eventually result in symptomatic and severe cases as a bilateral pneumonia and acute respiratory distress syndrome (ARDS) with associated histopathologic findings including exudative and proliferative phases of diffuse alveolar disease (DAD) that have the complicated form of COVID-19 [1, 2, 6, 8, 15, 16, 17, 18, 19]. Additionally, there are numerous comorbidities that increase the risk of severe cases of COVID-19, which includes hypertension, obesity, metabolic syndrome, diabetes, cerebro-cardiovascular disease and older age [6, 20].

Viremia (the presence of virus in the blood or circulatory system), in general, is only casually discussed in most scientific research papers as if in passing and there exist a paucity of references [21]. Additionally, the mechanisms of viremia are seldom discussed with any indepth mechanisms of how viruses enter the capillary pulmonary venous circulation and hence to the heart for systemic arterial delivery to the extrapulmonary target end-organs. Since the ACE2 enzyme is the entry receptor for the SARS-CoV-2 virus and is expressed in multiple extrapulmonary tissues and organs (Fig.3) it is important to discuss the process of viremia in greater detail as a plausible mechanism of injury to the host.

Once the virion SARS-CoV-2 daughters are shed from the Pn2 alveolar epithelial cells they accumulate within the alveolar air sacs and then enter the Pn1 alveolar epithelial cells where they severely damage and/or infect the Pn1 lining epithelial cells of the alveoli and are then subsequently transmitted to the Pn1 basement membrane (BM). From the BMs of the Pn1 cells the daughter virions then penetrate and breech the damaged fused BM with the fused BM of the abluminal alveolar capillary endothelial cells and progress to become located within the alveolar capillary endothelial lumen by either absorptive transcytosis (across) or paracellular transmigration (between) ECs to reside within the alveolar capillary lumen (Figs 1, 2). As the 
virion daughters pass these barriers of the BGB there will be marked dysfunction and damage to each of these respective cells previously discussed. For example, the damaged Pn1 and ECs would allow an easier pathway for the next successive waves of daughter virions due to the continued Pn2 alveolar invasions by the viral infection from the naso-oropharynx and upper airway respiratory epithelial cells by SARS-CoV-2 virus and daughter virions (Figs. 1, 2). Additionally, as the virus infects the Pn2 cells it decreases surfactant synthesis and excretion adding to alveolar dysfunction with alveolar collapse and also decreases the re-epithelialization of the alveolus of the Pn1 cells, since the Pn2 cells also act as the precursor cell and are responsible for Pn1 re-epithelialization when damaged.

Viremia has even been detected by positive reverse transcription polymerase chain reaction (RT-PCR) of blood plasma for the SARS-CoV-2 virus at least in one patient and known to have occurred two days prior to the onset of symptoms of dry cough and fever and diagnoseable by RT-PCR nasopharyngeal swabbing in a highly immunocompromised patient [22]. Therefore, viremia may be an even earlier finding in the COVID-19 pandemic and further supports the important link between known pulmonary infections and the involvement of the extrapulmonary target organs. As previously mentioned, there are very few papers discussing viremia in the literature and it has been suggested that SARS-CoV-2 nucleic acid testing in the blood may be a marker for rapid deterioration of COVID-19 pneumonia to demonstrate a high viral load in those with positive test results [21].

The importance of virus/virion load or viral/virion "storm" due to uncontrolled replication of the SARS-CoV-2 in the respiratory epithelium, plasma and/or Waldeyer's ring nasal respiratory epithelium as it relates the development of critical COVID-19 is not to be underestimated. Importantly, Bermejo-Martin et al, were able to demonstrate that viral RNAemia (via RT-PCR measurements in 250 swab-positive human patients) was markedly associated with critical illness and a dysregulated host response [23]. They demonstrated activation of endothelial cells and a host of inflammatory changes suggesting an hyperimmune response or cytokine storm and increased thromboembolism via increased D-dimer (a fibrin degradation product and is named after two D fragments of the fibrin protein joined by a crosslink upon fibrinolysis) in COVID-19 human patients [23]. To summarize, this group demonstrated that the SARS-CoV-2 viral load of viremia was associated with critical illness, endothelial dysfunction, hypercytokinemia, systemic inflammatory response, tissue damage, neutrophil activated responses and coagulation activation. Furthermore, they concluded that RNAemia and viral RNA load in the plasma could be utilized as possible marker to not only assess a response to treatment but to add to the capability of predicting disease outcome $[23,24]$. Additionally, the viral load (RNAemia) appears to be closely associated with elevated level of interleukin 6 (IL-6) in those patients who are critically ill with COVID-19 [25].

\section{Viremia: The Lymphatic System}

The lymphatic system may be characterized by having primary organs consisting of the bone marrow hematopoietic stem cells and the thymus (involution with aging) and the secondary organs of the lymphatic system including the tonsillar system or Waldeyer's ring (WR) (Fig. 4), lymph nodes and the mucosa-associated lymphoid tissue (MALT) interspersed within WR. Specifically, the nasal associated lymphoid tissue (NALT), the bronchial-associated lymphoid tissue (BALT) and the gastrointestinal tract commonly referred to gut-associated lymphatic system (GALT) or also known as Peyer's patches are also included in these secondary organs 
[26]. Even though the tonsillar system of Waldeyer's ring is considered a secondary organ of the lymphatic system, it acts as the first line of defense against the invading SARS-CoV-2 virus. Additionally, WR forms an incomplete circle of lymphatic tissue encompassing the nasal and oral routes for incoming air to be eventually delivered to the lungs via the orderly progression from the naso-oropharynx to the larynx, trachea, bronchi, bronchioles, terminal bronchioles, alveolar ducts, alveolar air sacks and the BGB [6, 26, 27, 28]. Importantly, the posterior nasal respiratory mucosa region is currently the gold standard for specimen collection to clinically diagnose COVID-19 via nasopharyngeal swabs by reverse transcription polymerase chain reaction (RT-PCR) because of the higher concentration of ACE2 receptors being located more posteriorly (Figs. 4, 5) [29].

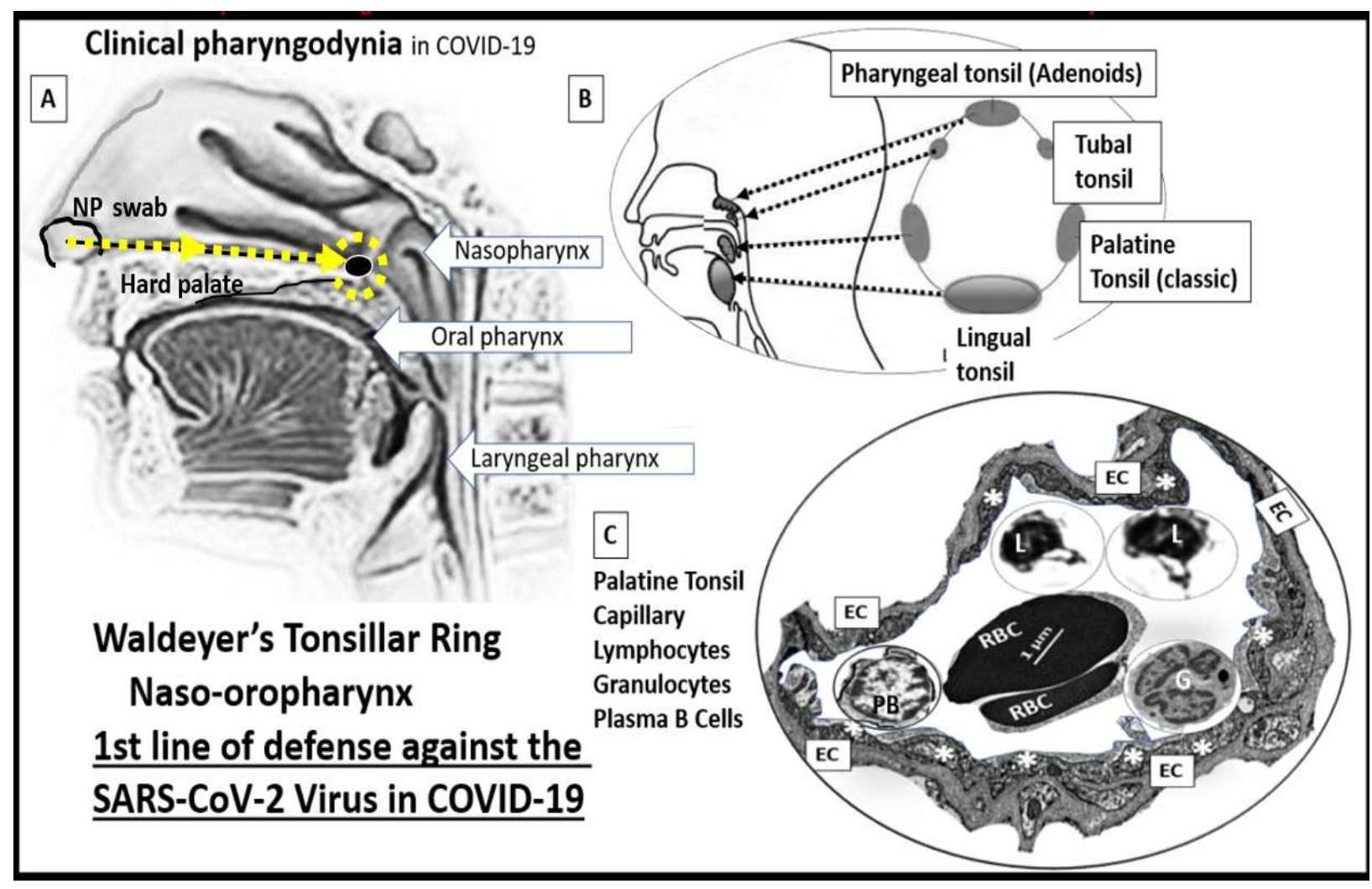

Figure 4. Clinical pharyngodynia, Waldeyer's ring, and tonsillar capillary of the vascular and lymphatic systems. This image demonstrates a sagittal illustration of the head and neck emphasizing the nasooropharynx as the gold standard for diagnosing COVID-19 (yellow dashed line) (A) [30]. Waldeyer's ring of the naso-oropharynx secondary lymphatic system (B) and a transmission electron microscopic image of a tonsillar capillary with representative red blood cell(s) (RBCs), lymphocytes (L), granulocytes $(G)$ and cells with the nuclear chromatin pattern suggestive of a plasma B cell (PB) within the endothelial lining cells (EC) in the vascular system (C). A lymphatic system tonsillar capillary is not shown and do not contain RBCs. The naso-oropharynx has become the preferred tissue to be globally sampled for the clinical diagnosis of COVID-19 (yellow dashed line in (A). Recently, the amount of genomic viral load at the time of admission to hospital from this diagnostic site has been demonstrated to be independently associated with the outcomes for intubation and ventilator assisted breathing and mortality. Importantly, the viral load from this region was found to be predictive even above and beyond advanced age and other comorbidities, which makes this clinical anatomical site of great importance [31]. 
The secondary lymphatic system of Waldeyer's ring is the initial point of entry by the SARS$\mathrm{CoV}-2$ virus. Here, the virus S1 spike protein interacts with ACE2 receptor located on the surface of the respiratory epithelium mucosa cells covering the lymphatic tissues of WR. The ACE2 receptor with its serine protease transmembrane protease serine 2 (TMPRSS2) invades the epithelial cell via fusion with its bilipid layer plasma membrane and taken up by these cells via the endocytic pathway utilizing the Furin protease plus acidic $\mathrm{pH}$ and other endocytic proteases. This process allows the SARS-CoV-2 virus to take over and exploit the synthetic machinery of the host cell to undergo replication and produce increasing numbers of SARS-CoV-2 daughter virions and eventual endocytic secretion and expulsion of daughter virions leaving behind a damaged and dying cell. These necrotic/apoptotic-pyroptotic cells trigger pathogen- or damageassociated molecular patterns (PAMPS/DAMPS) to activate the toll-like receptor systems to result in activation of the innate and the subsequent inflammatory adaptive immune systems.

Herein, it is important to note that sore throat - pharyngodynia is now one of the listed presenting symptoms of COVID-19 introduced around May 2020 along with the new symptom of loss of taste or smell along with nasal congestion or runny nose by the CDC and the WHO $[32,33]$. Additionally, there have been case reports, which demonstrated swelling and enlargement of WR lymphatic tissues by quantitative measurements with magnetic resonance imaging studies [34]

Therefore, the clinical history and examination of the ear nose and throat and neck associated with symptoms may be important findings when considering COVID-19 since ear, nose and throat (ENT) symptoms of new onset of loss of smell and taste along with sore throat and lymphadenopathy may be more common that currently discussed in the existing literature (Figs. $4,5)[33,3435,36]$. Both innate and adaptive immunity are known to occur in the nasooropharynx NALT tissue of the nasal mucosa (Fig. 4) [26]. Additionally, adaptive immunity is also known to be initiated in the secondary lymphoid organs such as the spleen and lymph nodes that are initiated by the innate immune system (Figs. 4, 5) [35 37]. Kaneko et al were able to demonstrate that there was an absence of geminal centers and even loss of Bcl-6 germinal center B cells in lymph nodes in patients with severe COVID-19 infections at autopsy [35, 37]. These findings in the lymph nodes correlate with the known loss of white pulp in the spleen [35, 3736 38]. 


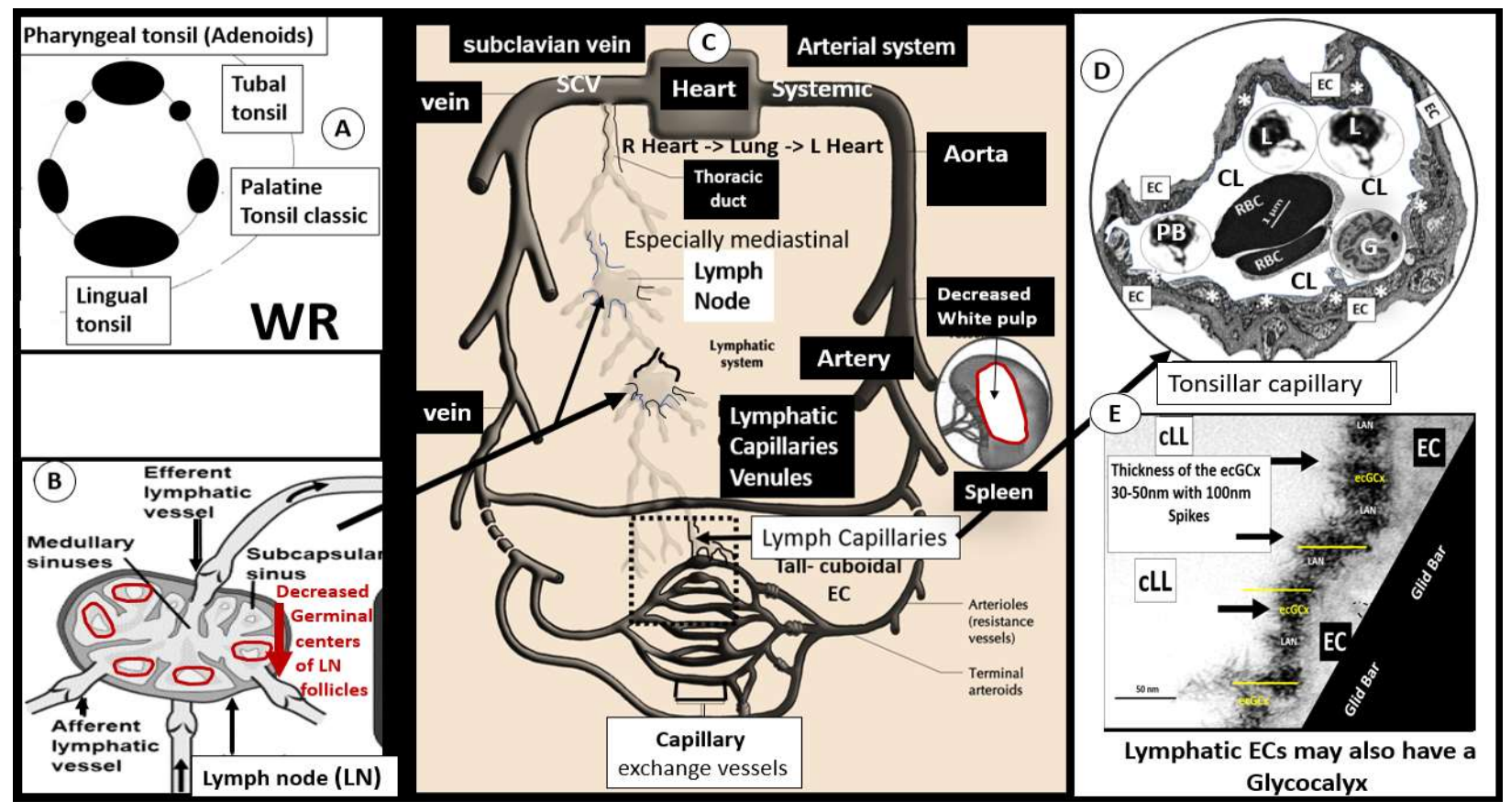

Figure 5. Each of the lymphatic nodes of Waldeyer's ring (WR) including the nasopharyngeal, tubular, palatine and lingual tonsils are capable of being infected by SARS-CoV-2 virus. Panel A illustrates Waldeyer's Ring (WR) and is the point of initial infection by COVID-19 - SARS-CoV-2 virus and this lymphatic ring is the first line of defense that the virus has to overcome. It is here that the virus binds to the ACE-2 receptors and gains entry and infects these naso-oropharyngeal respiratory mucosal cells with viral replication and thus provides the possibility for infecting others via coughing sneezing or even breathing with aerosol droplets distribution to become capable of infecting others. This implicates the protective function of mask wearing to prevent the spread of aerosol transmission. Importantly, the localized infection within WR may be the first to involve viremia since the lymph vessels drain into regional cervical lymph nodes of the lymphatic system as depicted in panels $\mathbf{B}$ and $\mathbf{C}$ and hence to the lymphatic thoracic duct to be distributed via the systemic circulation in order to promote the earliest phase of viremia and also may implicate early splenic infection. Importantly, the virus replicates within these respiratory epithelial mucosal cells and moves to upper respiratory tract epithelial cells and progresses to the lower respiratory tract epithelial pneumocyte cells of the AVU and BGB pneumocytes and AVU-BGB endothelial cells to develop direct pulmonary derived viremia discussed in previous sections 1 and 2 . Panel D is a representative depiction of a tonsillar capillary. Panel E illustrates that the lymphatic ECs may also be lined by an endothelial glycocalyx that may be attenuated and or lost as a result of shedding or collapse due to infection by the SARS-CoV-2 virus especially in those patients with T2DM (control C57B15 female model stained with lanthanum [LAN]). In addition to the removal of an excess fluid transport that leaks from physiological capillary exchange in the capillary beds of peripheral organs and tissues the lymphatic vessels are important for immunosurveillance and even immunomodulation whenever there is pathogen involvement such as the SARS-CoV-2 viral infection $[37,39]$. CL = capillary lumen; $c L L=$ capillary lymphatic lumen; $E C=$ endothelial cell; $e c G C x=$ endothelial glycocalyx; $G=$ granulocyte; $L=$ lymphocyte; $L A N=$ lanthanum nitrate staining for glycocalyx; $P B=$ plasma B cell.

The novel betacoronavirus, SARS-CoV-2 shares 79\% genome sequence identity with the original severe acute respiratory syndrome coronavirus (SARS-CoV of 2002-2003) and of the 
four structural genes, SARS-CoV-2 shares more than $90 \%$ amino acid identity with SARS-CoV except for the spike S gene, which diverges and may allow for the high infectivity of COVID-19 [40]. Because of this similarity one might follow the path of viral transmission from Waldeyer's ring of the lymphatic system into the draining lymph nodes as described in Chinese rhesus macaques (Macaca mulatta) and also in human patients by Liu et al. [28]. It is important to note here that in both humans and macaques the lymphatic system drainage to the cervical lymph nodes occur from naso-oropharynx and Waldeyer's ring while trachea and lung lymphatics drain to the tracheobronchial and hilar mediastinal lymph nodes, respectively. These authors found by immunohistochemistry that all experimental macaques infected with SARS CoV by nasal inoculation were positive for viral nucleoprotein (NP) and viral ribonucleic acid (RNA) into the Waldeyer's ring tonsil and MALT as compared to non-infected controls. Additionally, all draining lymph nodes tested contained the NP antigen, which was in agreement with viral seeding into the entire respiratory mucosal tissues within two days post infection. The spleen proved to be less frequent as compared to the lymph nodes at two days post infection; however, by seven days post infection all spleens were positive. These results suggested that within two days following infection that infected cells quickly disseminated the SARS CoV virus to draining lymph nodes and that systemic dissemination occurred not only in the spleen but also the small intestines by day seven post infection [28]. As a result of this study one can possibly translate this data to the very similar SARS-CoV-2 virus associated with COVID-19 in humans which has yet to be accomplished in current studies. However, we do know that the coronaviruslike particles have been indentified in multiple extra-pulmonary tissues demonstrating systemic viral dissemination [41].

While nasopharyngeal swabs are currently the standard of care [42] to test for the presence of COVID-19, salivary testing is currently evolving and may be available for consumer use in the near future [43].

\section{Endothelium}

The vascular endothelium is an autocrine, paracrine and endocrine organ that is essential for maintaining vascular tone and homeostasis $[6,44,45,46,47]$. The endothelial monolayer of cells $\left(\sim 7,000 \mathrm{~m}^{2}\right)$ with its intact endothelial glycocalyx and functional endothelial nitric oxide synthase (eNOS) enzyme allow it to become an interface between the blood compartment and the immediate surrounding tissues. Additionally, the endothelium acts as a gatekeeper or sentinel of the vessel wall, which in the quiescent or healthy state provides for the functional control of vasomotion, oxidative-redox stress, inflammation, vascular permeability and structure [46].

\subsection{Endothelial Activation and Dysfunction}

The term endothelial activation was initially coined and characterized by Willms-Kretschmer et al [48] who noted that ECs became plump-thickened (ultrastructural change by electron microscopy) and also leaky (functional change) as they studied delayed hypersensitivity reactions in 1967 and was further defined on a more functional basis in 1988 by Prober who was able to demonstrate that EC activation can produce dysfunction with or without injury $[49,50]$ (Fig. 6). 


\begin{tabular}{|c|c|c|c|}
\hline $\begin{array}{l}\text { Non-activated Quiescent EC } \\
\text { Control - healthy }\end{array}$ & \multirow{2}{*}{ 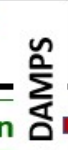 } & \multicolumn{2}{|c|}{$\begin{array}{l}\text { Activated EC in T2DM and SARS-CoV-2 } \\
\text { Infected Cells and Downstream effects }\end{array}$} \\
\hline 1. Anticoagulant / Anti-aggregation & & Procoagulant / Pro- & -aggregation \\
\hline $\begin{array}{l}\text { Thrombomodulin Prostacyclin } \\
\text { Intact ecGlycocalyx - heparans }\end{array}$ & $\check{\Sigma}$ & $\begin{array}{l}\text { Tissue Factor; Thromboxan } \\
\text { Neutrophil NETS (neutroph }\end{array}$ & $\begin{array}{l}\text { e; CD40; Erythrocyte adhesion } \\
\text { hil extracellular traps); platelet activation/adhesion }\end{array}$ \\
\hline 2. Pro-fibinolytic & & Anti-fibinolytic & \\
\hline UPA and TPA & & PAI-1 & \\
\hline 3. Anti-inflammatory & & Pro-inflammatory & Leukocyte attraction/adhesion \\
\hline $\begin{array}{l}\text { Intact ecGlycocalyx; prevents leukocyte } \\
\text { Interactions flow and Nitric oxide (NO) }\end{array}$ & 은 & $\begin{array}{l}\text { ecGlycocalyx attenuation a } \\
\text { cellular adhesion molecule } \\
\text { Interactions of flow and } \mathrm{Ni}\end{array}$ & $\begin{array}{l}\text { and/or loss; promotes } \\
\text { es VCAM ICAM; Chemokines (MCP-1) } \\
\text { itric oxide anti-inflammatory }\end{array}$ \\
\hline 4. Barrier Function intact & & Barrier Function & attenuated and/or lost \\
\hline Adherens Junction; VE-Cadherins intact & $\frac{u}{\sim}$ & $\begin{array}{l}\text { Attenuation and/or loss of } \\
\text { Attenuation and/or loss, sh } \\
\text { EC lifting, separation, sloug }\end{array}$ & $\begin{array}{l}\text { VE-Cadherins. BM remodeling thickening degradation } \\
\text { eedding of ecGlycocalyx } \\
\text { ghing, Senescence, EC apoptosis }\end{array}$ \\
\hline 5. Vasodilation & & Vasoconstriction & \\
\hline $\begin{array}{l}\text { NO; Prostacyclin; EDHF } \\
\text { (Endothelium-derived hyperpolarizing factor) }\end{array}$ & 는 & Decreased bioavailable $\mathrm{NO}$ & ; Thromboxane \\
\hline 6. Anti-oxidant & & Pro-oxidant & \\
\hline $\begin{array}{l}\text { Superoxide dismutase (SOD) } \\
\text { Glutathione (GSH) } \\
\text { Heme-oxygenase (HO-1) }\end{array}$ & 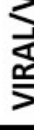 & $\begin{array}{l}\text { NADPH Oxidase } \\
\text { Oxidases; superoxide }^{-} \\
\text {RONS - (reactive oxygen nit } \\
\text { Peroxynitrite - } \mathrm{ONOO}^{-}\end{array}$ & trogen species); \\
\hline
\end{tabular}

Figure 6. Comparison of non-activated healthy controls and activated endothelial cells as occurs in type 2 diabetes and COVID-19 infected individuals with the SARS-CoV-2 virus. This comparison highlights the differences between the normal healthy quiescent control endothelium with essential components outlined in green boxes on the left-hand side of the figure. Each of these essential components is compared to the activated and dysfunctional endothelium outlined in red boxes on the right-hand side of this figure. It is interesting to note that nuclear factor kappa B (NF- $\kappa \mathrm{B})$ is activated as a result of glucotoxicity and AGE/RAGE interaction and these multiple pro-oxidant mechanisms associated with redox storm and viral load of SARS-CoV-2 virus [51]. This activation subsequently incites further proinflammatory mechanisms and also contributes to a vicious cycle of ongoing EC activation and associated EC dysfunction. Activated and dysfunctional endothelial cell(s) (ECs) in type 2 diabetes mellitus (T2DM) will be capable of having a detrimental synergistic effect when infected with SARS-CoV-2 virus in COVID-19. $B M=$ basement membrane; ec = endothelial cell; $E C=$ endothelial cell; $C D-40=$ cluster of differentiation-40; ICAM = intercellular adhesion molecule; $M C P-1=$ monocyte chemoattractant -1 ; NADPH = nicotinamide adenine dinucleotide phosphate; PAI-1 = plasminogen activator inhibitor; $U P A=$ urokinase plasminogen activator; TPA= tissue plasminogen activator; $V E=$ vascular endothelial; $V C A M=$ vascular cell adhesion molecule.

Even though EC activation may exist in a spectrum of changes some have outlined types I and II activation. EC activation type I does not require de novo protein synthesis or gene upregulation and occurs rapidly with EC retraction, the expression of P selectin and release of von Willebrand factor. EC activation type II takes longer to allow the stimulating agent such as the SARS-CoV-2 virus to allow for gene activation and transcription with protein synthesis that involves adhesion molecules, cytokines and tissue factor $[49,50]$. Importantly, there are at least five core changes in EC activation, which include (i) loss of vascular integrity with increased permeability; (ii) increased expression of leukocyte adhesion molecules including E-selectin, ICAM-1 and VCAM-1, which allows leukocytes to adhere to endothelium and then move into the tissues (Fig. 
7); (iii) phenotype change from antithrombotic to prothrombotic with loss of surface anticoagulant molecules thrombomodulin and heparan sulphate of the ecGCx and decreased fibrinolysis due to increased plasminogen activator inhibitor (PAI-1), increased platelet adhesion and aggregation and tissue factor; (iv) increased cytokine production such as interleukins -1, -6 that regulates the acute phase response, tumor necrosis factor alpha ( $\mathrm{TNFa})$, monocyte chemoattract protein-1 and (v) upregulation of human leukocyte antigen complex encoded by major histocompatibility complex molecules $[49,51]$. It is important to note that EC activation is not only a spectrum of morphological and functional changes but also when one is viewing ECs with transmission electron microscopy it may be observed that some ECs may be retracted or lifted from the intima while adjacent ECs may appear to be morphologically normal and additionally, there may be an abrupt swelling of ECs when studying the EC monolayer even in diseases that are known to have EC activation and dysfunction (personal observations) [52].

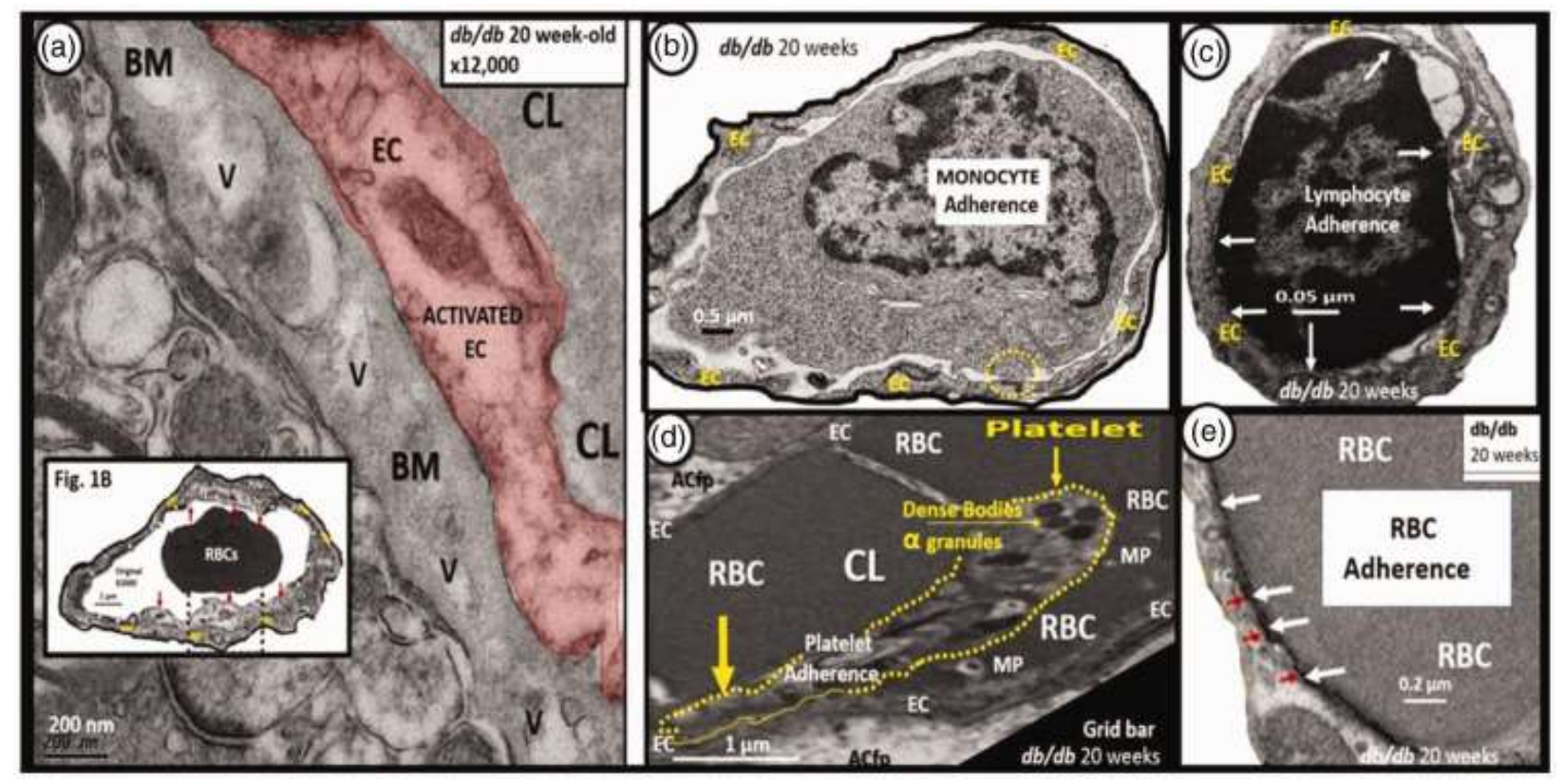

Figure 7. EC activation, dysfunction and vulnerable endothelial cell(s) (ECs) in patients with type 2 diabetes mellitus (T2DM) and COVID-19. This multi-panel image depicts endothelial cell (EC) activation with leukocyte, platelet and erythrocyte adhesion from cortical brain tissue layer III in a 20week-old female diabetic mouse $(\mathrm{db} / \mathrm{db})$ with obesity, insulin resistance and type 2 diabetes mellitus (T2DM). Panel (a) note the plump thickened EC (pseudo-colored red) and the thickened vacuolated (V) basement membrane (BM) and the insert at lower magnification in panel a. Panel (b) note the adherence of a monocyte and a lymphocyte (panel c) in addition to an adherent platelet (panel d outlined by yellow dashed line) and an erythrocyte - red blood cell (RBC) (arrows depicting electron dense adherence plaques between $\mathrm{RBC}$ and $\mathrm{EC}$ ) in panel (e). This image is provided by $\mathrm{CC} 4.0$ [6]. Images have varying magnifications and scale bars. $C L=$ capillary lumen; $M P=$ microparticles.

\subsection{The Endothelial Cell Glycocalyx (ecGCx)}

The highly polarized EC acts as a gatekeeper and sentinel barrier to protect vascular integrity against increased permeability, inflammation and thrombotic insults. It is capable of synthesizing at least three prominent protective barriers, which include: (i) basolateral cell-cell adherens junctions, which consist of vascular endothelial cadherin(s) (VE-Cadherins) that bind 
the EC cytoplasmic domains of proteins (p120, $\beta$-catenin, plakoglobin and cytoskeletal actin assembly proteins) to adjacent ECs creating a paracellular barrier [53]; (ii) the basilar plasmalemmal extracellular matrix (ECM) basement membrane (BM) [54] and (iii) the apicalluminal sugar-protein coated ECM of the ecGCx as a permeability barrier of the endothelium that is dependent on size and sieving mechanisms, electrostatic charge and selective EC plasma membrane cell receptors (Fig. 8) [55].

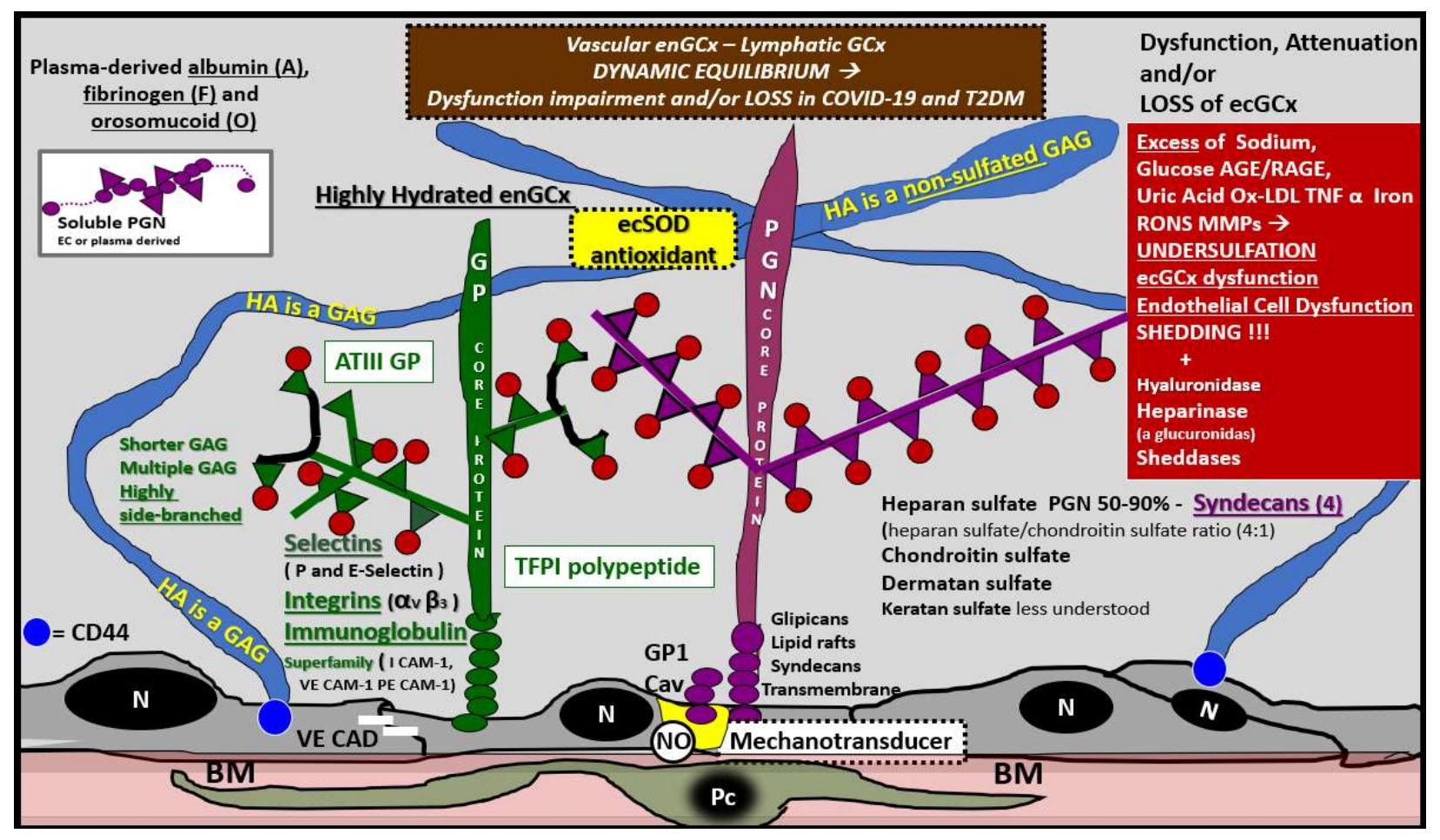

Figure 8. Endothelial glycocalyx (ecGCx) in type 2 diabetes mellitus (T2DM) and COVID-19 is attenuated and/or lost. This illustration is a simplified depiction of the highly complex ecGCx and depicts the three major anchoring proteins of the ecGCx: proteoglycans (1) (purple), glycoproteins (2) (green) and hyaluronan (3) (blue). The ecGCx is in a constant state of flux, being constantly resynthesized, and is highly compressible by the blood's cellular constituents (red and white blood cells). The proteoglycan(s) (PGN) are noted for their syndecans (plus others) and the glycoproteins are noted for their selectins such as intercellular adhesion protein, P-selectin and E-selectin. Note the glycosaminoglycans, long linear disaccharides of proteoglycans (purple triangles), and shorter highly branched glycosaminoglycan oligosaccharides of the glycoproteins (green triangles) that are highly sulfated (red dots). This surface layer has a net negative charge (along with additional orosomucoid proteins) and opposes movement of negatively charged molecules in the blood to the underling endothelium. This size selective semipermeable surface barrier (hindering access of proteins $70 \mathrm{kDa}$ or larger) additionally serves as a glucose and sodium sink that can be rapidly overcome with attenuation and/or shedding in diabetes or clinical conditions resulting in excessive sodium chloride. The intact ecGCx contains a protective extracellular superoxide dismutase (ecSOD) and antithrombin glycoprotein III (ATIII) while serving as a mechanotransducer as a result of utilizing the glycosylphosphatidylinositol (GPI) anchoring glypicans within the caveolae (Cav) lipid rafts (yellow indentation) and endothelial nitric oxide (NO) synthase enzyme. The ecGCx varies from 100 to $500 \mathrm{~nm}(300 \mathrm{~nm}$ mean) in thickness and from 1 to $2 \mu \mathrm{m}$ in length. The percentage of ECs covered by the ecGCx was approximately $40 \%$ in the capillaries as shown by transmission electron microscopic studies and was greater in the brain as compared with the heart or lung lacking a blood-brain barrier. In our cortical brain transmission electron micrographs using lanthanum nitrate staining we found a minimum base coating of $50 \mathrm{~nm}$ with thickness up to 100 to 200 
$\mathrm{nm}$ (see Figure 10) possibly caused by multiple dehydration steps. Glucotoxicity, oxidative stress and neuroinflammation (as in the diabetic $d b / d b$ model), traumatic shock injuries, ischemia/reperfusion and sepsis can result in shedding the ecGCx and/or a reduction in its volume and plasma levels of syndecans, intercellular adhesion molecules, selectins and hyaluronans. The PGN perlecan is decreased in the BM of patients with diabetes in renal tissues and high glucose impairs the permselectivity based on loss of anionic charge.

The ecGCx was initially identified over $1 / 2$ Century ago by JH Luft in 1966 who utilized transmission electron microscopy to examine the highly positive charged cationic stain ruthenium red [56]. Since the review by Pries et al. in 2000 regarding the endothelial surface layer [57] there has been an exponential increase in the number of publications and were up to 178 in the year 2020. Currently, the cationic stain lanthanum nitrate has been shown to be a reliable and reproducible staining method by author and others and is gaining respectable momentum $[58,59]$. In fact, Inagawa et al. have demonstrated the reliability of the stain in the microcirculation of not only the pulmonary microcirculatory capillaries but also in the brain [58, 59] and author has also demonstrated its usefulness in demonstrating the elusive ecGCx in control models and its attenuation and/or loss in diabetic cortical, hippocampus, midbrain and choroid plexus brain capillaries in the novel female diabetic leptin deficient BTBR $o b / o b$ cortical brain (unpublished studies) $[6,54,60]$. Underlying comorbidities such as T2DM, obesity, hypertension and cerebro-cardiovascular diseases are known to result in ecGCx remodeling with attenuation $[6,61]$. When there is dysfunction, attenuation, damage, shedding or loss of the ecGCx protective barrier there would be an easier access to spike protein attachment and entry of the SARS-CoV-2 virus spike protein into the vascular endothelium ACE2 receptor that might create a vicious cycle of increased viral-virion load with associated endothelial activation and dysfunction (Fig. 9). 


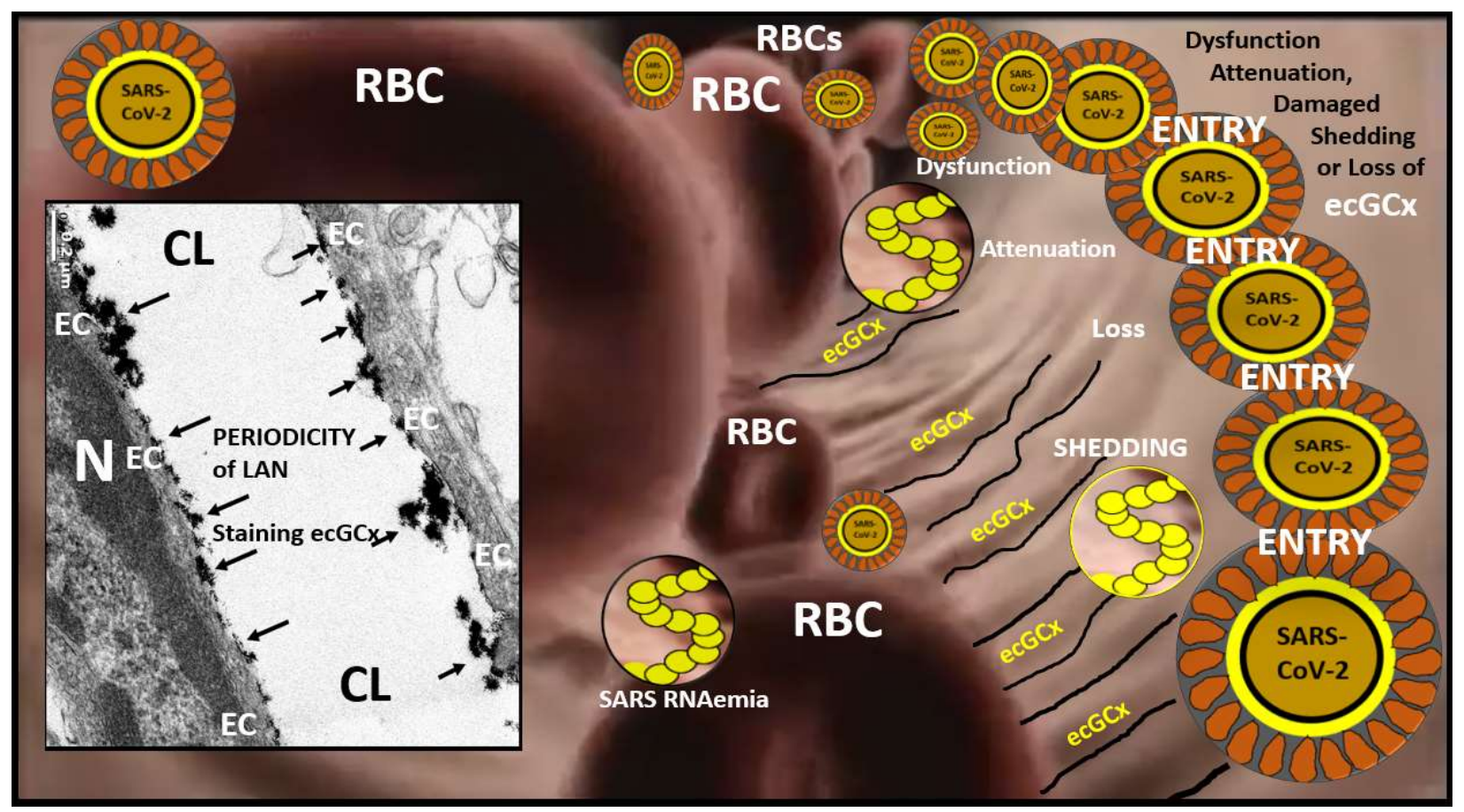

Figure 9. Dysfunction, attenuation, damage, shedding and/or loss of the endothelial glycocalyx (ecGCx) may result in an increased attachment and entry of the SARS-CoV-2 virus into endothelial cell(s) (ECs) with activation. This image depicts an intravascular view of a very small arteriole or capillary as can be noted by the single line of red blood cell(s) (RBC) entering this microvessel. Note that on the right $1 / 2$ of this image there are ruffles suggestive of the periodicity of the protrusions of the endothelial glycocalyx (ecGCx) and how to the far-right these ruffles are absent and the vascular wall is smooth. Additionally, note the insert on the far left-hand side of this image, which depicts a transmission electron micrograph (TEM) of a lanthanum nitrate (LAN) perfusion fixed hippocampal continuous capillary with multiple periodic protruding electron dense staining of LAN to demonstrate the periodic nature of the protruding ecGCx (arrows) [62]. The SARS-CoV-2 virus and its ribonucleic acid (RNAemia) are represented and illustrate the increased entry into the regions, which have sustained attenuation and loss of the ecGCx. These changes suggest that if the exGCx is perturbed that there may be increased entry of the virus and thus perpetuate the SARS-CoV-2 viremia with increased entry into the endothelium with associated EC activation and dysfunction with a proinflammatory and prothrombotic surface. This background image is not to scale; however, the inset TEM image has a magnification $\mathrm{x} 10,000$; scale bar $=0.2 \mu \mathrm{m}$. CL $=$ capillary lumen.

COVID-19 primarily affects the pulmonary system with the SARS-CoV-2 viral infection resulting in severe acute respiratory syndrome (SARS) of acute respiratory disease (ARD) with diffuse alveolar damage histopathologic changes. The SARS-CoV-2 virus is capable of causing EC activation and dysfunction and act synergistically with underlying pre-existing comorbidities such as T2DM. The important role of an intact and functioning ecGCx barrier is not to be underestimated and plays a key role in the development and progression of COVID-19 with increased morbidity and mortality $[62,63]$. Knowledge is still accumulating in regards to the protective functions of the ecGCx as it applies to not only the integrity of EC functions but to each and every cell.

Even though there has been an explosion in knowledge regarding the ecGCx, there remains a great deal of information about this fascinating structure and its functional importance and the 
author is currently very interested in the detrimental effects of "undersulfation" of glycosaminoglycans and its role in the dyshomeostasis of the ecGCx not only in T2DM but also COVID-19.

\section{Circulating Blood Cells}

\subsection{Erythrocytes - Red Blood Cell(s) (RBCs)}

Mammialian RBCs have evolved to become anuclear and lack cellular organelles in order to be dedicated primarily to the delivery of life-sustaining oxygen and removal of the metabolic byproduct carbon dioxide via iron containing hemoglobin $(\mathrm{Hb})$ and an intact vascular system [64]. Additionally, RBCs, platelets and fibrin(ogen) are essential for hemostasis, coagulation and instigating wound repair. RBCs have a constant turnover and removal and it is estimated that two million newly formed RBCs (of a total $\sim 25$ trillion) enter the circulation and are cleared by the reticuloendothelial system in the liver and spleen every second [65]. RBCs appear as biconcave discs wherein the outer rim contains most of the Hb molecules and the inner dimple reflects the absence of the nucleus creating a central indentation (Fig. 10).

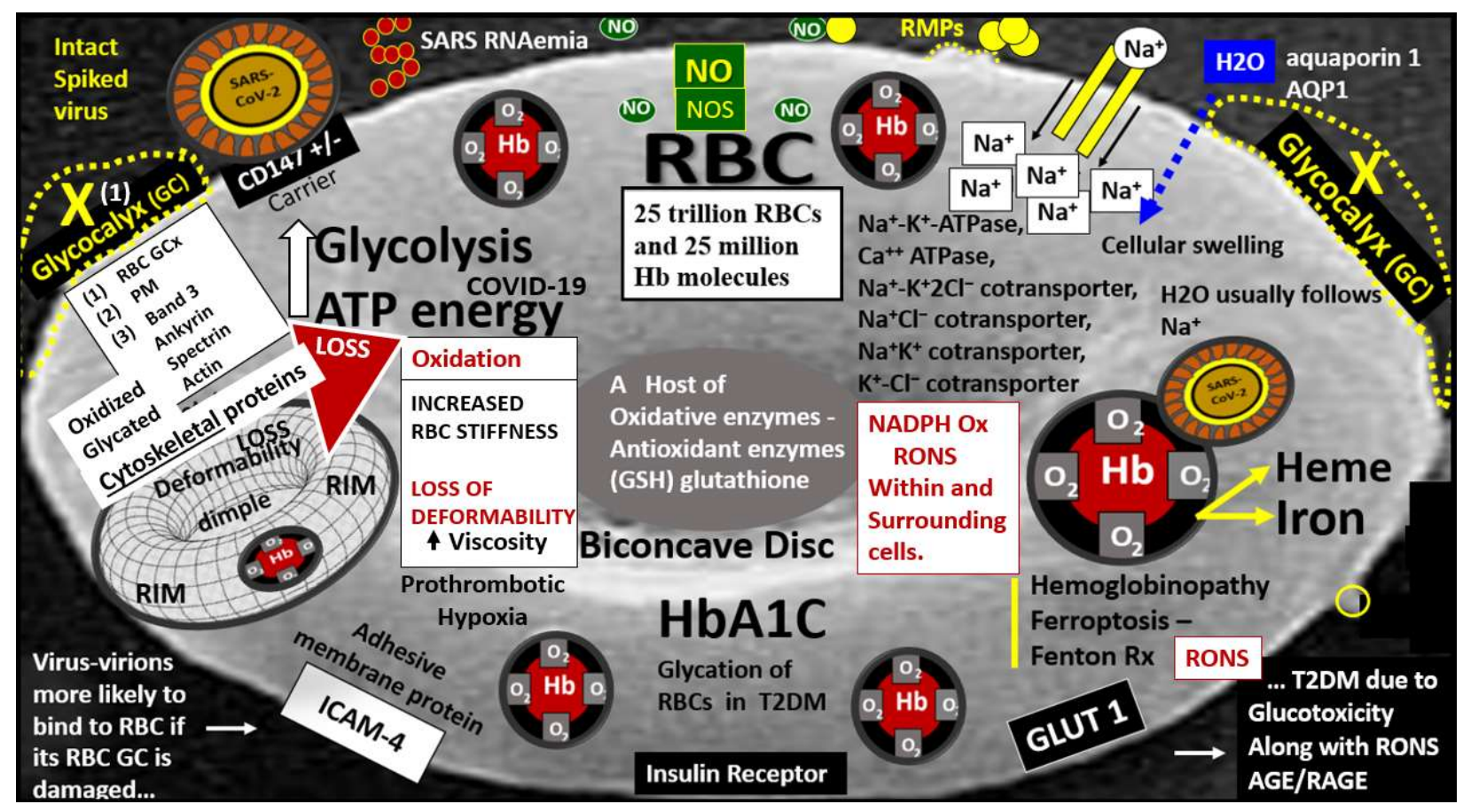

Figure 10. RBC transmembrane proteins and remodeling. This image is of a single RBC that has been enlarged to illustrate some of the interesting transmembrane proteins and multiple interesting facts and figures which facilitates visually what is discussed within the text. Importantly the interaction between the SARS-CoV-2 virus, nuclear proteins (RNAemia) and/or its spike proteins with either the RBC glycocalyx or its unproven CD147 or ACE2 receptor - ACE2 interacting proteins might promote RBC carrier transport assisting transfer of the virus throughout the vascular system. The virus will result in the dissociation of heme and iron from hemoglobin $(\mathrm{Hb})$ with an increase in redox stress and the underlying redox storm within the lumen to further increase reactive oxygen nitrogen species (RONS). The cumulative effects of T2DM and COVID-19 will accelerate the loss of deformability of RBCs and the increase in redox stress-storm and hemoglobinopathy associated with COVID-19 with pre-existing T2DM. Once the RBC is altered either morphologically (decreased deformability) or metabolically there 
will develop impaired rheology, sludging - stalling at capillaries and perturbed viscosity in addition to increased clotting - immunothrombosis. RBC decreased deformability, margination of platelets to activated ECs, phosphatidylserine (PS) flipping from cytosol to luminal bilipid layer increase EC adhesion, RBC prothrombotic microparticles (RMPs), which scavenges NO and release of ADP and thromboxane A2 activates platelets and all promote accelerated thrombosis. There are at least 50 or more well described transmembrane proteins that primarily deal with either transport or adhesion. Note that the RBC has three distinct portions of its outer membrane: (1) RBC glycocalyx (RBC GCx); (2) plasma membrane and (3) the band 3, ankyrin, spectrin and actin cytoskeleton network to support deformability. This illustration is not to scale and therefore no scale bar was inserted. ATP = adenosine triphosphate; $A D P=$ adenosine diphosphate; $\mathrm{Ca}++=$ calcium; $\mathrm{Cl}$ - = chloride; $\mathrm{CD} 147=$ cluster of differentiation Basigin (BSG) - extracellular matrix metalloproteinase inducer (EMMPRIN); GLUT 1 = glucose transporter 1; HbAIC = hemoglobin A1C; ICAM-4 = Intercellular Adhesion Molecule-4; $\mathrm{K}^{+}=$ potassium; $\mathrm{Na}+=$ sodium; $N O=$ nitric oxide; $N O S=$ nitric oxide synthase; $R B C=$ red blood cell(s); $R M P=$ red blood cell microparticles; $T 2 D M=$ type 2 diabetes mellitus.

Metabolically, RBCs provide their energy via the glycolytic pathway to produce adenosine triphosphate (ATP). They have an outer RBC glycocalyx (Figures 8) and a bilipid layer plasma membrane similar to other cells; however, the composition and structure of the RBC glycocalyx has not been adequately studied to know how it might differ or be affected by various stressors as compared to the more extensively studied ecGCx in section 4.2. For example, when studying the capillaries and RBCs in the hippocampal brain regions of the diabetic, obese leptin deficient diabetic BTBR $o b / o b$, author has been able to observe that the ecGCx of the endothelial cells are attenuated and/or lost while the RBC glycocalyx remains to stain positive when utilizing a perfusion fixed lanthanum nitrate staining protocol, which indicates that they these two glycocalyces may have differences in remodeling changes when exposed to this diabetic milieu (Fig. 11). Indeed, more studies are recommended to study the composition and structure of the RBC glycocalyx in the future especially in those affected by COVID-19.

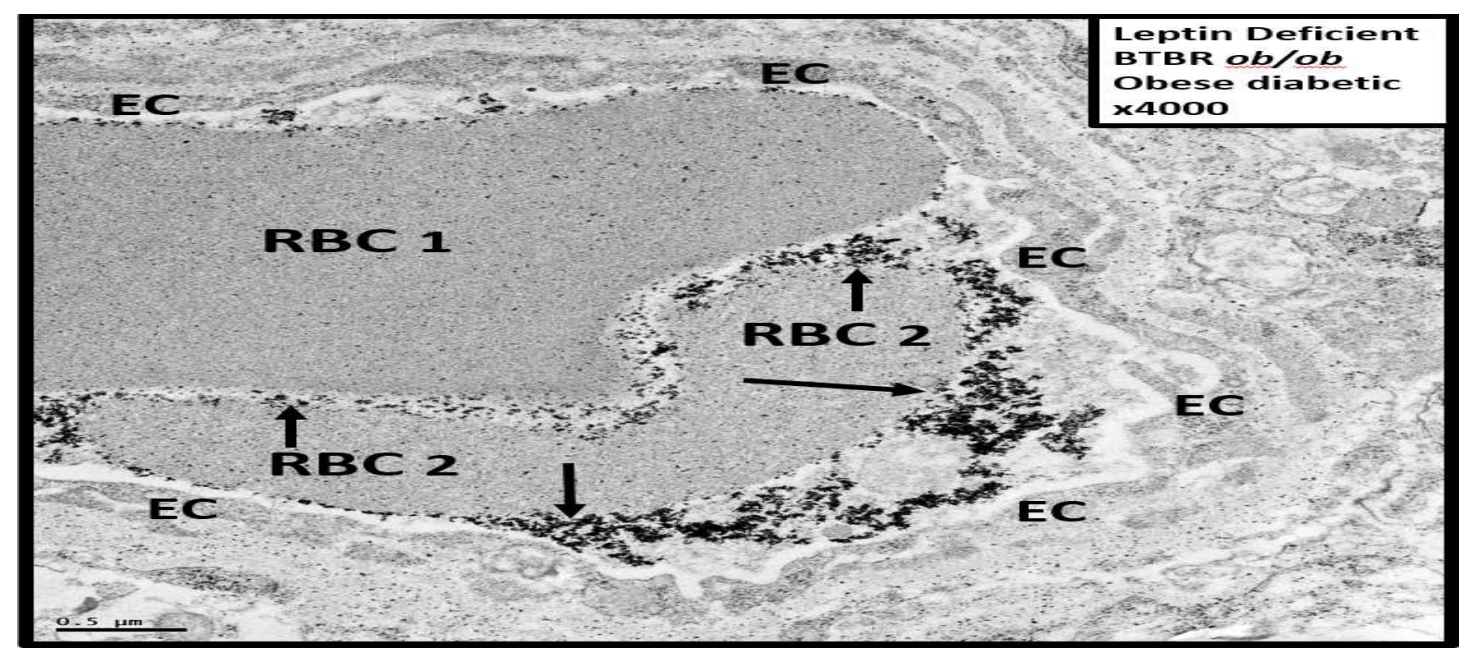

Figure 11. Red blood cell glycocalyx intact in female diabetic hippocampus with concurrent endothelial glycocalyx shedding in a diabetic preclinical model. Note that the red blood cells (RBCs) are heavily decorated by the electron dense lanthanum nitrate perfusion fixation stain for the glycocalyx while the endothelial cell glycocalyx has undergone a complete loss or shedding of its glycocalyx. This image illustrates that the RBC glycocalyx (arrows) may have a different composition or structure as compared to the endothelial cells glycocalyx, in that, the RBC glycocalyx was spared from undergoing loss or shedding under the influence of obesity insulin resistance and type 2 diabetes mellitus due to a genetic 
deficient leptin model in the BTBR ob/ob female mouse preclinical model at 26 weeks of age. Magnification x4000; scale bar $=0.5 \mu \mathrm{m}$.

RBCs have a tremendous strength of their plasma membrane provided via their cytoskeletal proteins to endure their required flexibility and folding (deformability) capabilities to traverse their smaller microcapillary systems and withstand the trauma of a highly pressurized, high velocity closed arterial blood flow system. RBCs have a life-span of $\sim 120$ days and as they become senescent or damaged, they are removed via phagocytosis in the reticuloendothelial system of the hepatic and the splenic sinusoids. Importantly, the RBC plasma membrane has at least 50 transmembrane proteins that support interactions between RBCs, endothelial cells, intraluminal leukocytes and platelets [66] as well as offending pathogens including viruses such as the SARS-CoV-2 virus of COVID-19. In particular, RBCs support transport function including band 3 anion transporter, aquaporin 1(water transport), Glut 1 (glucose and Ldehydroascorbic acid transporter, Kidd antigen protein (urea transporter), RhAG (gas transporter function), $\mathrm{Na}^{+}-\mathrm{K}^{+}$-ATPase, $\mathrm{Ca}^{++}$ATPase, $\mathrm{Na}^{+}-\mathrm{K}^{+}-2 \mathrm{Cl}^{-}$cotransporter, $\mathrm{Na}^{+}-\mathrm{Cl}^{-}$cotransporter, $\mathrm{Na}^{+}-\mathrm{K}^{+}$cotransporter, $\mathrm{K}^{+}-\mathrm{Cl}^{-}$cotransporter, Gardos Channel $(\mathrm{Ca}(2+)$-dependent $\mathrm{K}(+)$ efflux and of course the hormonal Insulin receptor [66]. Adhesive membrane proteins of the RBCs include ICAM-4, which interacts with integrins and Lu, the laminin-binding protein. There are multiple clusters of differentiation such as (CD36, CD 47, CD147, CD55 and CD59, and cellular adhesion molecule CD44).

Previously, RBCs were thought to not have a tissue-cellular RAAS nor an ACE2 enzyme to act as a receptor for SARS-CoV-2 virus attachment with its spike proteins. Therefore, some implicated that RBCs might respond to this virus via a CD147 receptor that might be important for SARS-CoV-2 binding and/or entry into the RBCs in patients with COVID-19. as a result of localization of CD147. SARS-CoV-2 spike protein (S1) was observed in SARS-CoV-2 infected Vero E6 cells by immuno-electron microscope $[67,68]$. However, this has recently come into question [69], while D'Alessandro et al. have shown by utilizing proteomics that angiotensin and ACE2-interacting proteins were present on RBC surface membranes [70]. Furthermore, this group suggested that even though the RBCs cannot support viral replication due to a lack of a nucleus and organelles that RBCs may theoretically be invaded by the SARS-CoV-2 virus and be directly or indirectly targeted by pathogens and possibly serve as a stealth carrier cell in viremia.

Once the SARS-CoV-2 virus or its RNA contents (RNAemia) have invaded and passed the multiple barriers of the BGB - AVU in pulmonary tissues along with its lymphatic delivery to the systemic circulatory system there will be immediate exposure of the virus and/or its nuclear proteins to roughly 25 trillion RBCs and 25 million $\mathrm{Hb}$ molecules [71]. With this early capillary and later systemic exposure of intraluminal RBCs to viremia or RNAemia the RBCs will have considerable exposure to the toxic virus and its contents, which allow for viral-RBC interactions. Certainly, there may be electrostatic and/or hydrophobic attractions between SARS-COV-2 virus and RBCs and recent reports implicates a stronger hydrophobic attraction between the two [72, 73]. However, since there is increased redox stress affecting the RBC due to increased glycolysis there may be a loss of electrostatic interaction making the RBC more vulnerable to intraluminal exposure.

The RBC has an outer RBC glycocalyx that can be identified as well as bilipid plasma membranes similar to other cells. Inserted into these plasma membranes are Band 3 with attachments to ankyrin, which is attached to alpha and beta-spectrin residues that eventually are 
attached to the cytoskeletal proteins (F-actin, tropomyosin and tropomodulin) and important for maintaining the shape, flexibility, durability, roughness, RBC distribution width and the deformability of RBCs (Fig. 12) [74]. In T2DM these skeletal membranes undergo remodeling of the Band 3 - ankyrin - spectrin actin membrane cytoskeleton and results in increased RBC stiffness with associated loss of deformability in T2DM. Loss of deformability of RBCs results in making the RBC quite vulnerable to further cytoskeletal membrane dysfunction and increase the loss of deformability not only when affected by T2DM but also the SARS-CoV-2 virus and viral RNA proteins $[72,74,75,76,77]$. When RBCs lose their capability to deform, they also lose their capability to squeeze through smaller capillaries with perturbation of RBC rheology, which is associated with increased viscosity, aggregation and thrombosis with possible thromboembolism. Furthermore, these changes result in sludging and stalling with increased hypoxia at the level of the capillaries that include both the pulmonary and extrapulmonary target end-organs of COVID-19 [6].

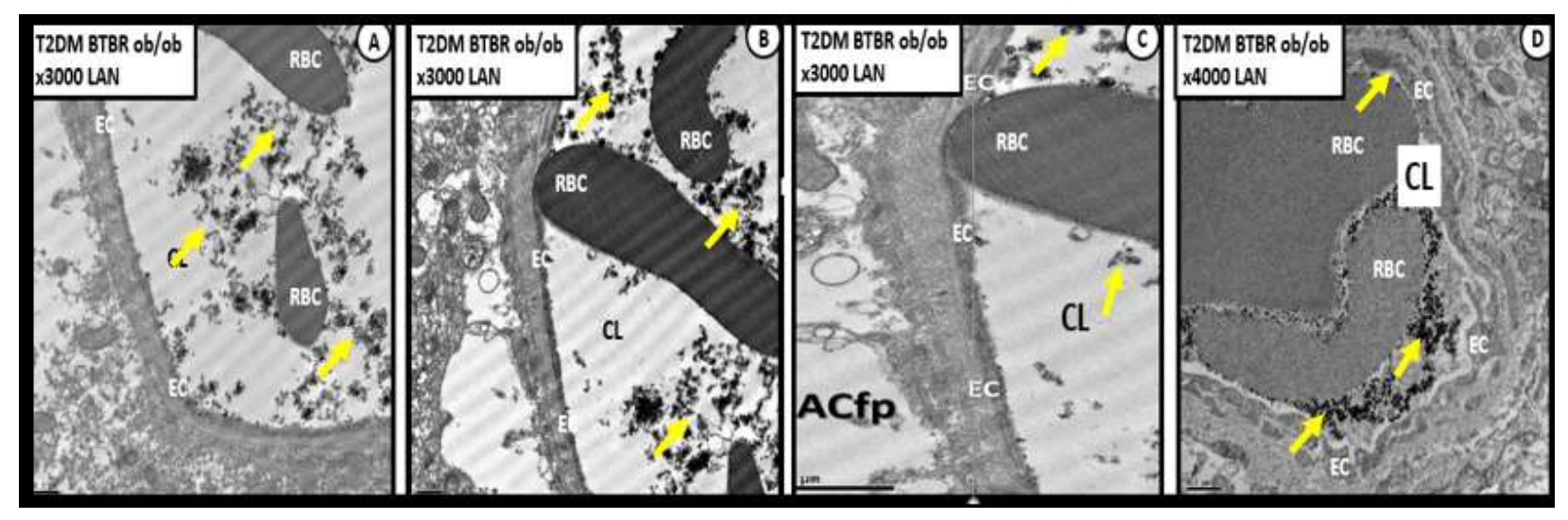

Figure 12. Red blood cell glycocalyx in T2DM diabetic model (BTBR ob/ob) with lanthanum nitrate. Note the intense electron dense staining of the red blood cell (RBC) glycocalyx (arrows) with lanthanum nitrate in this T2DM preclinical mouse genetic deficient leptin BTBR ob/ob model. Importantly, note that this model has near complete shedding of its endothelial cell glycocalyx (ecGCx). The presence of the RBC glycocalyx allows the RBCs to repel each other while there remains the possibility for the RBC to adhere to the endothelium due to its loss of the ecGCx. Importantly, note the interaction of the RBC in panels $\mathrm{B}, \mathrm{C}$ and $\mathrm{D}$. Also, our group has been able to image adhesion plaques between RBCs and the ECs in $\mathrm{db} / \mathrm{db}$ diabetic mouse models see figure 7(e). Magnification $\mathrm{x} 3000$ scale bar $=0.5 \mathrm{~nm}$ in panels A, B and $\mathrm{D}$ and $1 \mu \mathrm{m}$ in panel $\mathrm{C}$. While these representative neurovascular unit capillaries are from brain cortex layer III, others have demonstrated the presence of lanthanum nitrate (LAN) in pulmonary tissues that affect the endothelium as well as the RBC glycocalyx [58].

In addition to the loss of $\mathrm{RBC}$ deformability there is also an increase in $\mathrm{RBC}$ distribution width or anisopoikilocytosis (RBC variance in size and shape) which results in RBC removal by the red pulp sinusoids of the spleen $[72,74]$. RBCs have to constantly interact and communicate with one another, lining ECs, monocytes, lymphocytes, neutrophils, plasma proteins, pathogens and platelets. Furthermore, RBCs are intimately involved in the maintenance of thrombosis as well as being involved with immune response intravascularly and against pathogens [78]. The RBC glycocalyx (GCx) like the endothelial glycocalyx (ecGCx) is very important in both RBC function and structure and may be altered in not only T2DM but also COVID-19, which may act synergistically to increase multiple intraluminal perturbations such as increased thromboembolic events not only within the pulmonary systems but also the extrapulmonary target organs affected by COVID-19 [78]. The RBC GCx allows the RBC to interact with other cells that are found 
within the vascular lumen such as other RBC without adhesion due largely to the repulsion that is afforded to the RBC via its highly negatively charged GCx to repel other cells with their respective negatively charged glycocalyx such as other RBCs (decreased aggregation) EC and RBC adhesion, monocytes/macrophages, neutrophils and their neutrophil NETS or traps, lymphocytes and some intravascular plasma proteins [78].

RBCs contribute to thrombosis by their viscosity effects including stiffening and loss of deformation, margination of platelets to the vessel wall, platelet aggregation, flipping of phosphatidylserine from inner to outer bilipid plasma membrane and microparticles which support thrombin generation. Additionally, RBCs within thrombi stabilize and strengthens clot structure and impair fibrinolysis. In conclusion, RBCs are an important complement of the complex reactions of clot-thrombus formation.

\subsection{Thrombocytes - Platelets}

Platelets are classically known for their roles in hemostasis and pathological thrombosis along with the wound healing response to injury. Thrombotic complications in both the arterial and venous vascular beds are a major cause of morbidity and mortality in patients with COVID-19 [79]. Intravascular clotting or thrombosis due to thromboinflammation involves platelets, RBCs, fibrinogen-fibrin, thrombin and the coagulation cascade that is activated via inflammation (cytokine storm) and oxidative-redox stress-storm (RONS) that is tightly associated with an endotheliopathy (sections 4.1. and 4.2.), a hemoglobinopathy of the RBCs (section 5.1.) and the current section involving thrombocytopathy that eventually results in a coagulopathy with increased morbidity and mortality in COVID-19 [79].

Platelets are derived from megakaryocytes that are created from bone marrow hematopoietic stem cells, which develop cytoplasmic extensions of proplatelet elongations that pinch off and mature in the vasculature [80]. In COVID-19 it is now known that platelets become activated when the endothelium is damaged. Importantly, platelet activation is known to occur in T2DM $[6,46]$ and will be further activated when the ECs become damaged due to SARS-CoV-2 viral infection of ECs that further results in dysfunctional, activated and damaged ECs with a synergistic overall effect on EC dysfunction and activation (Fig. 13).

Platelet activation then associates with increased fibrinogen and fibrin to form fibrin platelet plugs when there is bleeding and platelet activation is very important function for hemostasis; however, it is detrimental when forming thrombi and chronically activated in relation to infarction of vessels and ischemia with tissue organ ischemia and cellular death.

Thrombocytopenia along with lymphopenia and elevated D-dimer has been a constant finding in patients with advanced COVID-19 and is known to be associated with increased risk and poor prognosis with increased morbidity and mortality [81]. There have been at least three possible mechanisms put forth regarding thrombocytopenia, which include the following: (i) SARS-CoV-2 may reduce platelet production due to cytokine toxicity to the bone marrow; (ii) SARS-CoV-2 infection may increase platelet destruction via toxicity of cytokines to bone marrow cells and inhibition of platelet synthesis; (iii) SARS-CoV-2 infection may increase platelet consumption due to extensive platelet aggregation in the lungs and extrapulmonary target end-organs [81]. While author favors platelet consumption it may be a combination of all three possible mechanisms. 


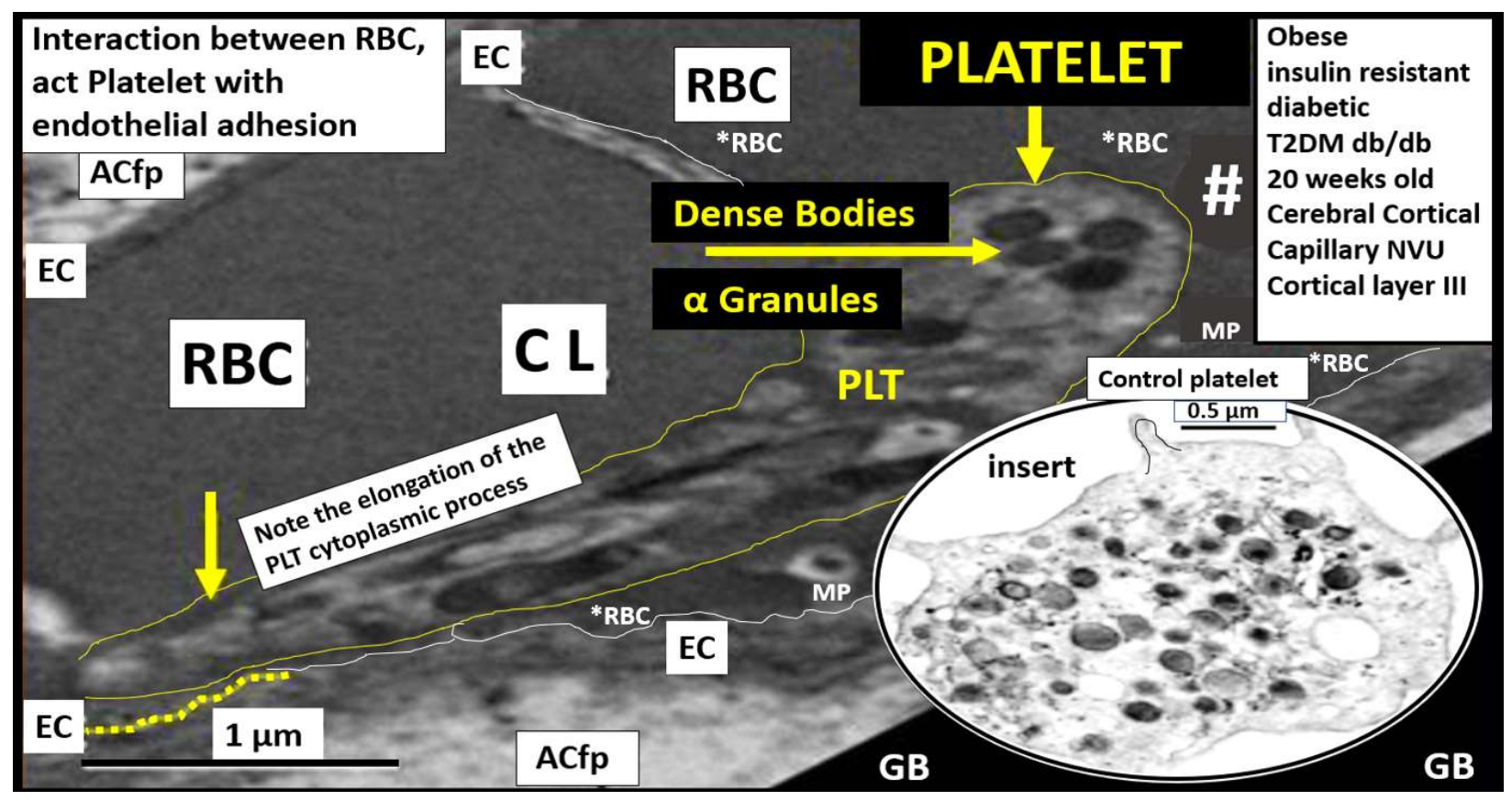

Figure 13. Activated platelet with adherence to an activated endothelium. This ultrastructural image depicts an activated (act) platelet (PLT) adhering to an activated cortical layer III capillary endothelium in a female type 2 diabetes mellitus (T2DM) mouse model ( $d b / d b$ at 20 -weeks of age). This activated PLT is outlined by a thin solid yellow line to contrast it from the surrounding red blood cells (RBCs) and note the yellow dashed line where there is adhesion to the activated endothelium of the endothelial cell (EC). This neurovascular unit capillary is from the cerebral cortex in cortical layer III. Not only is this activated platelet adhering to the activated endothelium but also note how it is interacting with the surrounding red blood cells in the formation of a microvascular thrombus. Note the long cytoplasmic extension of this act PLT as compared to the control non-activated platelet with only shortened cytoplasmic protrusions as observed in the insert image of a non-activated control platelet in the right lower region of this image. Also, note the $* \mathrm{RBC}$ outlined in white that is adjacent to the adherent activated platelet. Scale bar $=1 \mu \mathrm{m}$ in image and $0.5 \mu \mathrm{m}$ in the insert.

Viremia allows the SARS-CoV-2 virus or its nuclear contents (RNAemia) to be exposed to all of the blood compartmental cells of the circulatory vascular system including platelets. Platelets interact with each of the cells (RBCs, ECs, and neutrophils and their NETs - traps, monocytes and lymphocytes) and along with fibrin are known to have a cumulative effect on thrombosis with increased morbidity and mortality.

Activated platelets interact with leukocytes within the blood compartment by utilizing cellular interactions and juxtracrine signal via P-selectin and integrin $\alpha_{\mathrm{IIb}} / \beta_{3}$. Platelets are known to modulate leukocyte responses including migration, secretion, neutrophil extracellular traps along with the contribution to growing thrombi as well as triggering monocyte expression of tissue factor important for coagulation and thrombosis [82]. Therefore, platelet-leukocyte interactions are indispensable events in physiological and pathological hemostasis and inflammation.

5.3. Leukocytes - White Blood Cells: Mononuclear Monocytes and Lymphocytes and Granular Polymorphonuclear Neutrophils 


\subsubsection{Monocytes - Monocyte-Derived Macrophages - Resident Tissue Macrophages [Innate immune cells]}

Resident tissue macrophages (alveolar, interstitial and vascular monocyte-derived macrophages) reside in pulmonary AVU of the BGB and respond immediately to injurious stimuli such as the injury induced by the infection with the SARS-CoV-2 virus in COVID-19 [83]. Correspondingly, monocytes circulate along with SARS-CoV-2 virus, RBCs, platelets, neutrophils and lymphocytes within the intravascular lumen and lining ECs throughout the body once viremia has occurred. Thus, monocytes have the capability to reach each and every organ system and tissue. Specifically, monocytes serve as protective patrolling cells to aid in the phagocytosis of ongoing endothelial damage. Monocytes can be activated and signaled to exit the lumen primarily via endothelial paracellular routes when signaled by various chemokines including monocyte chemotactic protein $1(\mathrm{MCP}-1)$ and the cytokine granulocyte colonystimulating factor (G-CSF) at sites of tissue injury such as SARS-CoV-2 within the pulmonary alveolar vascular unit of the BGB (Fig. 14) [84].

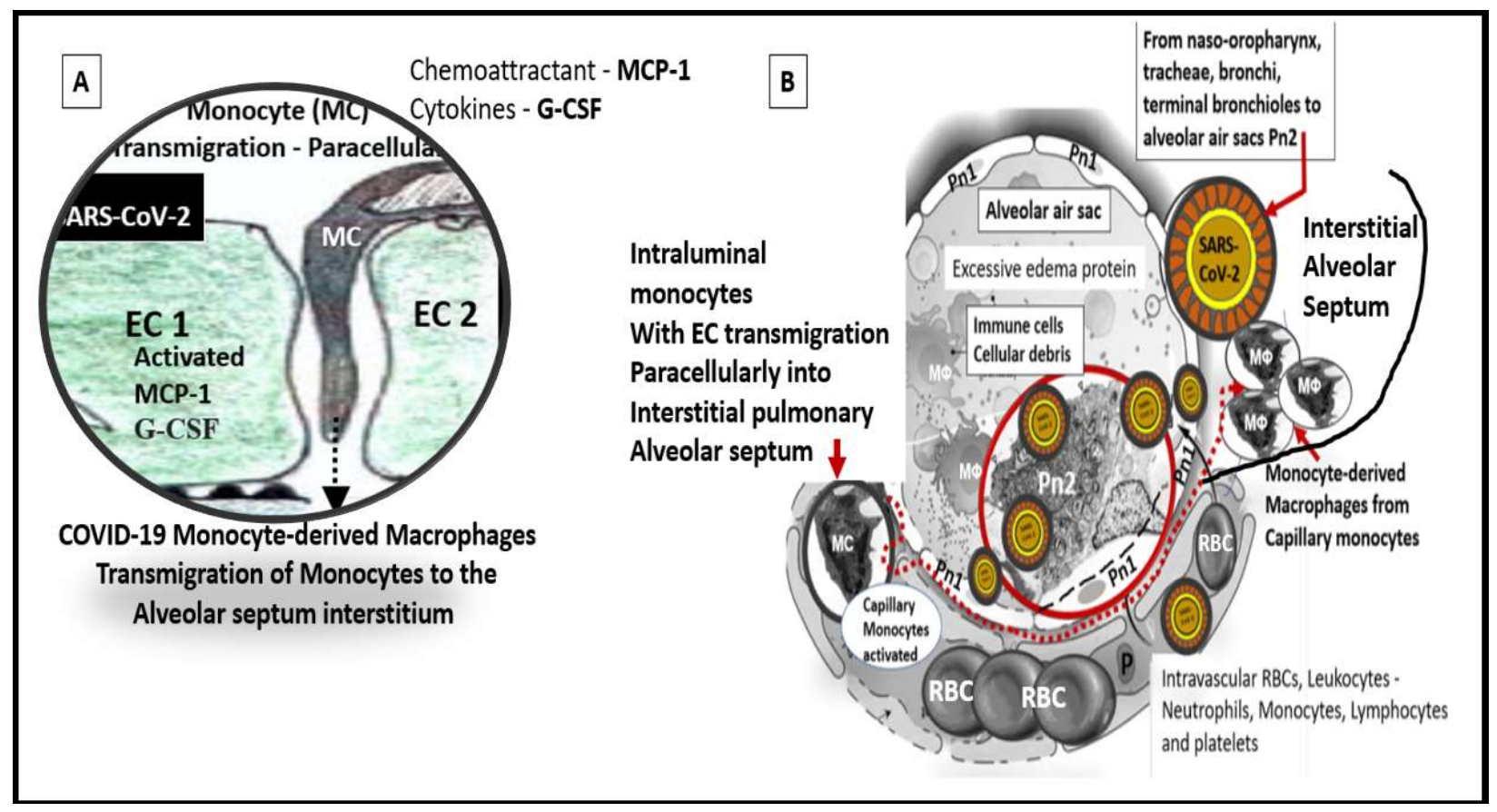

Figure 14. Monocyte transmigration to the alveolar interstitium in SARS-CoV2. (A) illustrates the monocyte (MC) undergoing active transmigration via the paracellular route as it is directed to the alveolar interstitium. (B) depicts the alveolar vascular unit of the lungs at the blood-gas barrier with an inflamed alveolar air sac with fibrinous exudate and an infected hypertrophic pneumocyte 2 (Pn2) with the SARS$\mathrm{CoV}-2$ virus. Note how the pneumocyte 1 (Pn1) cells line the entire alveolus with a thinned squamous like epithelium verses the cuboidal appearing Pn2 cell. Also, note the resident and monocyte-derived macrophages $(\mathrm{M} \Phi)$. This illustration demonstrates hyaline and fibrinous exudate within the alveolar air sacs and primarily interstitial $\mathrm{M} \Phi$. Interstitial and resident $\mathrm{M} \Phi$ s are usually more prevalent than intraalveolar MФ. EC = endothelial cell; $G-C S F=$ granulocyte colony - stimulating factor; $M C P-1=$ monocyte chemoattractant protein-1; $P n 1=$ pneumocyte $1 ; R B C=$ red blood cell.

Following this signaling and transmigration between adjacent ECs monocytes transform into monocyte-derived macrophages to aid in the phagocytosis of debris associated with viral 
infections and cellular loss via apoptosis - pyroptosis not only in the pulmonary BGB - AVU but also in the injured extrapulmonary tissues affected and infected by the SARS-CoV-2 virus.

Once these monocytes have transmigrated to the pulmonary alveolar septal tissue interstitium they have a propensity to become phenotypically polarized as either M-1 like (proinflammatory classically activated macrophages) or M-2 like (anti-inflammatory, healing - alternatively activated macrophages) $[84,85]$. During infections with SARS-CoV-2 virus the monocytederived macrophages are primarily of the polarized proinflammatory M-1 like macrophage types that are critical to the development of the "cytokine storm" or hyperinflammatory milieu of the intersititium that eventually becomes a systemic cytokine storm. Importantly, in T2DM the macrophages have already been signaled to become polarized M-1 like macrophages and these two diseases may be synergistic from the standpoint of monocyte-derived macrophages in the multiple extrapulmonary target end-organ when Covid-19 is presented with the co-morbidity of T2DM $[84,85,86]$.

While this section has examined the role of the monocyte/macrophage it is important to note that the monocyte/macrophage cells work closely together with neutrophil and extracellular traps and both help to orchestrate the power of the innate immune response system [87]. It is important to note here that neutrophils are not only capable of recruiting monocytes to becoming monocyte derived macrophages but also are capable of inducing the polarization of M2-like macrophage to the M-1like macrophage associated with infection of the SARS-CoV-2 virus.

\subsubsection{Neutrophils and Neutrophil Extracellular Traps (NETs) [Innate immune cells]}

Neutrophils (polymorphonuclear granulocytes) are considered to be the first leukocyte responders of the innate immune system to sites of the respiratory epithelium and pulmonary injury in a response to injury mechanism to pulmonary viral SARS-CoV-2 infections [88]. Generally, neutrophils are absent by light microscopic examination when autopsies or biopsies are performed with the macrophage and lymphocytes remaining the predominant cell types (Fig. 14). The sequence of the response to injury wound healing are outlined and include the acute and chronic inflammatory changes in this process in addition to the innate and acquired immune mechanisms in SARS-CoV-2 infections (Fig. 15). 


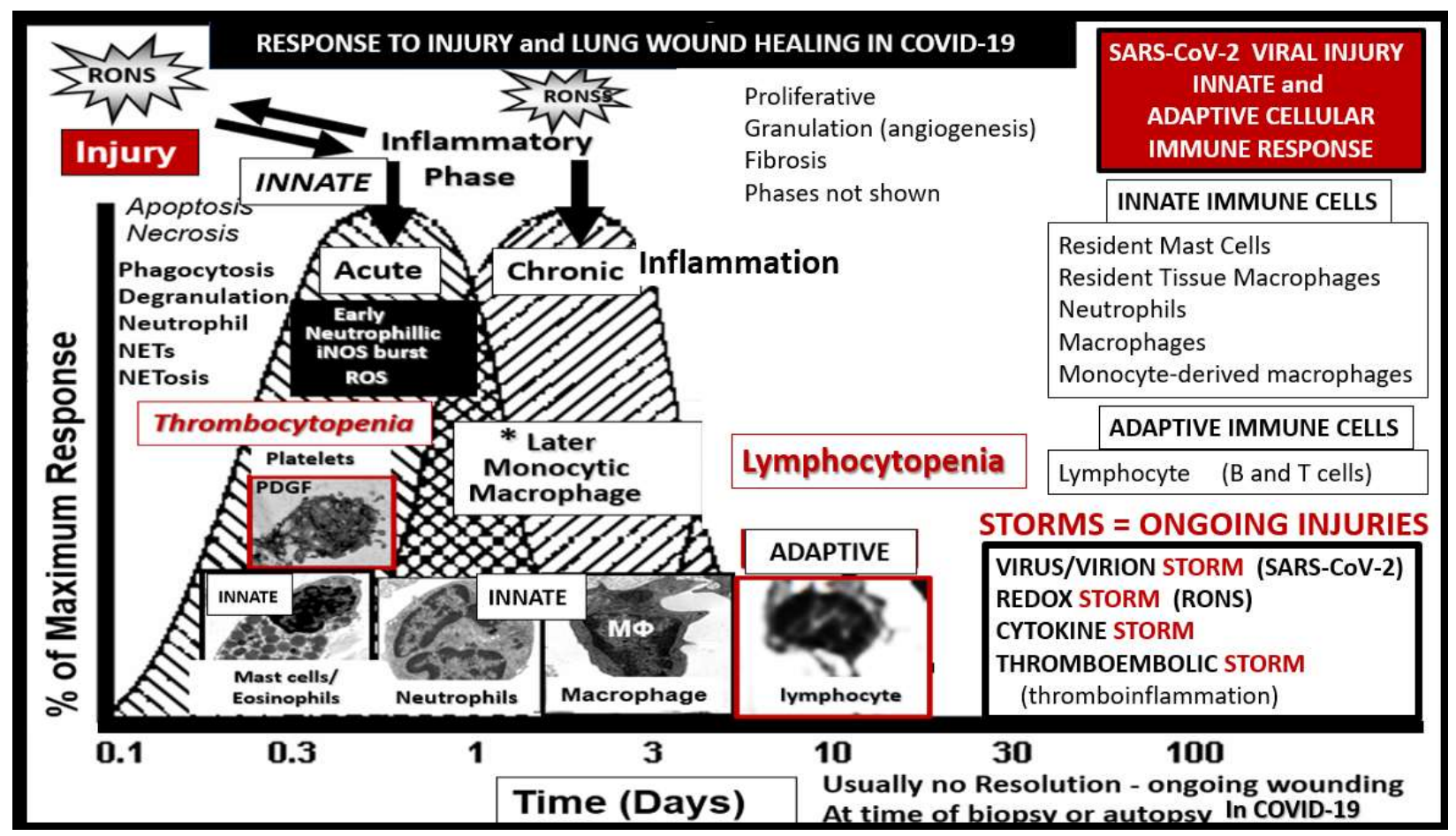

Figure 15. Response to SARS-CoV-2 injury and lung wound healing. This graphic illustration depicts the various cell types and their involvement in the response to injury to the SARS-CoV-2 virus as it invades the lung and also other extrapulmonary tissues. The innate immune cells consisting of innate mast cells - eosinophils and neutrophils (the first cells in the acute phase of inflammation), innate macrophages and the adaptive immune cell the lymphocyte (B and T types) are depicted. Note the thrombocytopenia and lymphocytopenia that are commonly found in those COVID-19 individuals with more serious disease requiring ICU and ventilator assisted respiration. Also note the importance of multiple storms in COVID-19, which represent ongoing injury to the pulmonary and extrapulmonary tissues and organs. The image was adapted with permission CC by 4.0 [6].

The granulocytic neutrophils respond to invading organisms such as the SARS-CoV-2 virus by phagocytosis and/or neutrophil extracellular traps (NETs) formation resulting in the process of NETosis. Both phagocytosis and NETosis (Fig. 16) involve an oxidative respiratory burst of prooxidant enzymes in neutrophils, which include NAPH Ox, myeloperoxidase, inducible NOS (iNOS) forming nitric oxide and peroxynitrite in addition to elastases [89]. 


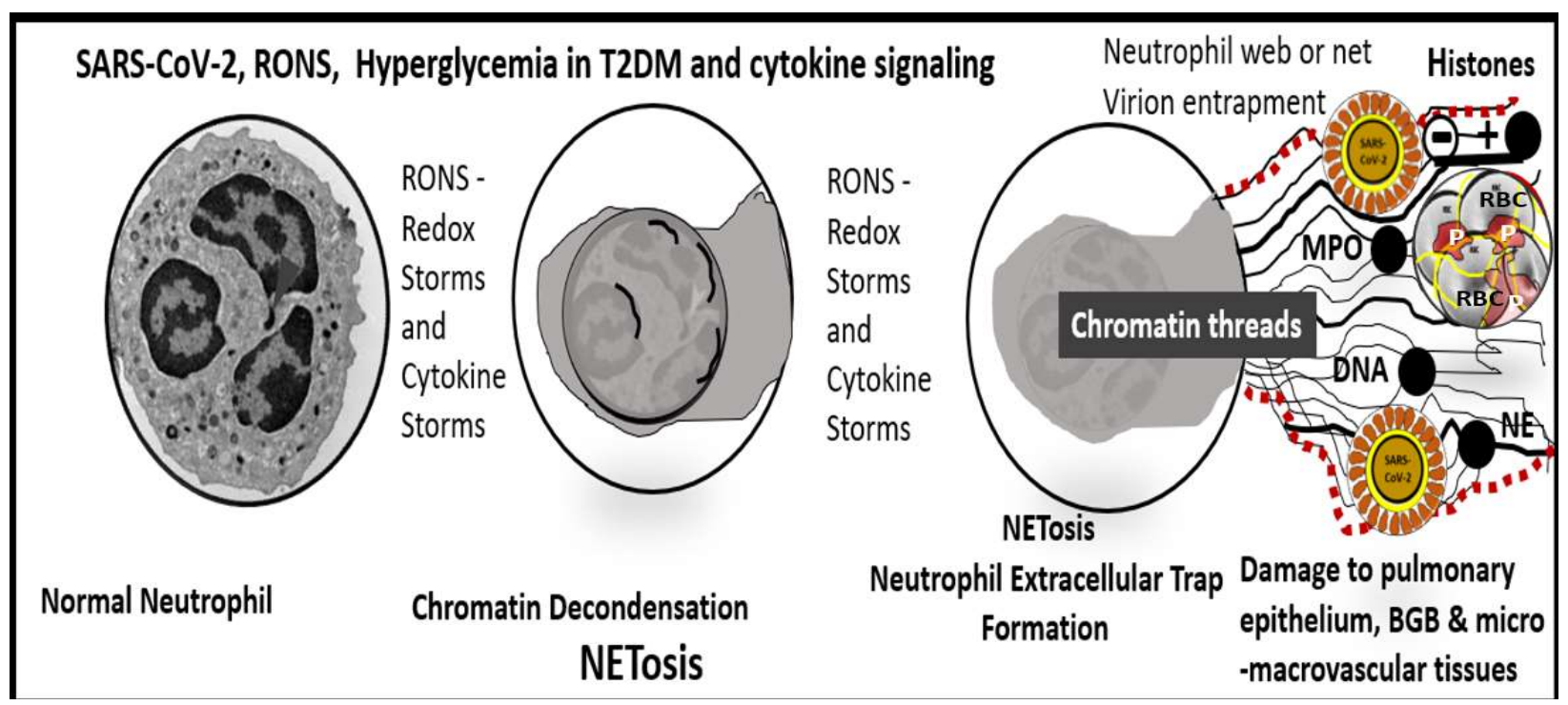

Figure 16. Neutrophil extracellular traps (NETs) and NETosis in T2DM and COVID-19. In T2DM and COVID-19. NETosis is detectable in circulating blood and associated with an inflammatory, prothrombotic state and impaired fibrinolysis. In addition to classic phagocytosis and degranulation as a part of the first response to invasive viruses the neutrophil is capable of forming neutrophil extracellular traps (NETS) to sequester pathologic viruses in a process termed NETosis. This process also supports the formation of thrombi as a result of platelets (P), fibrin (F) (yellow lines) and red blood cells (RBCs), which associate with impaired fibrinolysis in T2DM (upper right-had side of image). Note the electrostatic ionic binding between the citrullinated positive histones and the net negative charge of the SARS-CoV-2 viral particle(s) in the NET that assists in the entrapment of the virus. Also, note the initial essential step of nuclear decondensation of chromatin in the middle image that occurs prior to the release of chromatin threads in the far-right image. The following quotation seem applicable here: "Oh what a tangled web we weave, when first we practice to deceive" from Marmion: A Tale of Flodden Field by Sir Walter Scott indicating that excessive NETs - NETosis may be a double-edged sword with both pulmonary epithelial damage in the lung and intravascular cells with impaired rheology, vascular reactivity and the development of a vascular pro-constrictive and hypercoagulable intravascular milieu. $B G B=$ blood gas barrier; $D N A=$ deoxyribonucleic acid; $F=$ fibrin; $M P O=$ myeloperoxidase; $N E T=$ neutrophil extracellular trap; $N E=$ neutrophil elastases; $P=$ platelets; $R O N S=$ reactive oxygen nitrogen species; $S A R S-C o V-2=$ severe acute respiratory syndrome coronavirus 2; T2DM = type 2 diabetes mellitus.

It is commonly known that neutrophil migration and phagocytosis are impaired in diabetes and recently it has been noted that NETosis is increased in diabetes [90]. Furthermore, high glucose is associated with increased insulin resistance in COVID-19 [6.20] and may also contribute to increased NET formation along with increased cytokine hyperinflammation. NETosis and thrombosis, which includes thromboinflammation and thromboembolism [91].

\subsubsection{Lymphocytes [Adaptive - Acquired immune cells]}

The adaptive immune system and nongranular lymphocytes are the main cells found in lymph of the lymphatic system. Lymphocyte $\mathrm{T}$ cells in embryo and youth are created within the thymus and when the thymus undergoes involution in adulthood the $T$ cell population is capable of undergoing replication throughout its lifespan. The B cell lymphocyte is produced in the bone marrow from stromal cell precursors throughout life. The lymphocytes are transported within the lymphatic system and are sequestered in the lymph node germinal centers and the white pulp 
of the spleen and eventually drain via the thoracic duct into the subclavian venous system and enter the right atrium to proceed to the pulmonary circulation. Microscopically, they have a large nucleus and a thin rim of cytoplasm that may be characterized by three major types. The specific types of lymphocytes may be characterized by the T cells of (thymic origin, cellmediated and cytotoxic adaptive immunity that include the CD3+, CD4+ helper and CD8+ killer and $\mathrm{CD} 25+/$ FoxP3 + regulatory $\mathrm{T}$ cells), the B cells (of bone marrow origin, humoral, antibodydriven adaptive immunity) and NK cells (natural killer cells that differentiate and mature in the bone marrow, lymph nodes, spleen, tonsils, and thymus. The NK lymphocyte cells function in cell-mediated immunity and possess granules, cytotoxic innate immunity and importantly do not require prior activation in order to kill cells that may be missing the surface marker of the major histocompatibility complex (MHC class one) [27, 92, 93].

It is interesting to note that there may be some phenotypic subpopulations that vary from one organ to the other and even within the lung the phenotypic subpopulations of lymphocytes may vary at different points in time during their SARS-CoV-2 viral infections and their response to the severity of disease and viral load at the onset of the viral infection in COVID-19 [94, 95, 96, 97]. Furthermore, the SARS-CoV-2 virus may not only activate antiviral immune responses but also may be related to the uncontrolled inflammatory responses characterized by marked proinflammatory cytokine release syndrome or cytokine storm as discussed in section 5.1. This excessive immune reaction (cytokine storm) is strongly associated with lymphopenia, which is a constant finding in SARS-CoV-2 with diffuse alveolar dysfunction in COVID-19, lymphocyte dysfunction, and monocyte, granulocyte abnormalities as discussed in sections 5.3.1. and 5.3.2. respectively. In regards to the lymphocyte phenotypic subpopulation variations there seems to one constant and key finding in COVID-19 patients infected with the SARS-CoV-2 virus, that being lymphopenia and the associated increase in the neutrophil/lymphocyte ratio that has become a key and persistent finding in those seriously infected patients requiring intensive care unit due to increased morbidity. Importantly lymphopenia and the increased neutrophil/lymphocyte ratio has become a risk marker for increased morbidity and mortality during this pandemic $[27,28,98,99]$.

It is important to note that the viremic exposure of the SARS-CoV-2 virus to the acquired immune systems lymphocytes does not come without a price to pay, in that, those patients with the most severe cases resulting in death who have undergone autopsies have demonstrated not only an atrophy of the white pulp in the spleen but also a loss in the lymph node germinal centers which are certainly associated with the severity of the COVID-19 pandemic [37]. While these authors point to the importance of the loss of Bcl-6 expressing T follicular helper cells that may be caused by the marked cytokine hyperinflammation, the mechanisms of these atrophic and lost storage centers for lymphocytes have not been completely understood at this current time of the pandemic. These post-mortem autopsy findings certainly help to explain in part the consistent finding of lymphopenia that seems to be a central finding due to the SARS-CoV-2 virus of COVID-19.

\section{Conclusion}

This review has outlined in greater detail the possible sequence of events and mechanisms of viremia and its impact on the intravascular cellular milieu in the process of viral transmission and infection of the extrapulmonary target organs that are known to be infected by SARS-CoV-2 virus in COVID-19. The primary organ of infection (the pulmonary - alveolar vascular unit - 
BGB) and concurrent viremic intravascular cellular milieu, extrapulmonary target organs involvement and the combined comorbidities of T2DM and COVID-19 will greatly impact the increased morbidity and mortality. For those who wish to examine the process of viremia in general with greater depth and not specifically related to the SARS-CoV-2 virus the following reference is highly recommended [100].

Most epidemic and pandemic outbreaks can be traced to zoonotic infections that are transmitted from animals to humans. Some of these outbreaks might have been prevented by better hygiene, proper water sources and waste management and regular inspections of wild animal populations. However, others can be traced to the disturbance of ecological equilibriums or alterations to the environments in which pathogens habitually reside. This concept rings true yet today as set forth in a play entitled: Doctors Dilemma by George Bernard Shaw (1906) in which he wrote "The characteristic microbe of a disease might be a symptom instead of a cause". It is possible that we might invest in ecological and environmental conditions in order to prevent any future epidemics or pandemics. The combination of science and technology has certainly allowed our knowledge to prosper through this synergism during this COVID-19 pandemic. However, we still may be lacking in our examination of the microbe (SARS-CoV-2 virus) being a symptom rather than the cause in addition to the health disparities amongst those who are in minority situations around the world. These disparities include a multiple of variables influencing health, which includes physical environment, economic stability, ethnic and racial discrimination, education, access to nutritious food in terms of our social and community context, and importantly access to health care services [101]. We who ignore the past such as the 1918 Spanish flu pandemic are likely to repeat it and currently it appears that the COVID-19 pandemic is more closely paralleling the 1918 Spanish flu pandemic [6].

During this COVID-19 pandemic we have been introduced to the relatively novel so-called term "cytokine storm" in order to aid our understanding of the function of a dysregulated immune system - cytokine-release syndrome as a result of an overactivation of innate immunity and lymphopenia [102] and the excessive production of proinflammatory cytokines to result in a hyperimmune state associated with the hypercytokinemia that is associated with SAR-CoV-2 viral infection.

Throughout this review author has attempted to demonstrate that there are multiple storms that co-exist in this pandemic, which includes a viral/virion storm or viral load, a redox storm with excessive reactive oxygen-nitrogen species and a thromboembolic storm with excessive thrombosis resulting in ischemia not only within the pulmonary system but also in the extrapulmonary target organs in both the arterial and venous vascular systems that result in multi-organ system failure and mortality.

Frequently, throughout this review, author has pointed to the co-morbidity of pre-existing T2DM and how the interactions between the metabolic syndrome, obesity, insulin resistance and T2DM interact to increase morbidity and mortality in COVID-19 (Fig.17). Certainly, when one examines the co-morbidities associated with the more severe complications of COVID-19 there seems to emerge a solid core of at least five co-morbidities, which include (i) diabetes, (ii) hypertension, (iii) cerebro-cardiovascular disease, (iv) obesity and (v) older age. Importantly, when one examines the metabolic syndrome it is noted that there are at least four core variables present, which points to an important role of the co-morbidity of the metabolic syndrome as set forth by Reaven some three decades ago as syndrome $\mathrm{X}$ that was later renamed by Grundy two 
decades ago [103, 104]. Both the metabolic syndrome and COVID-19 have multiple variables and vulnerabilities and certainly COVID-19 has unmasked many of these vulnerabilities.

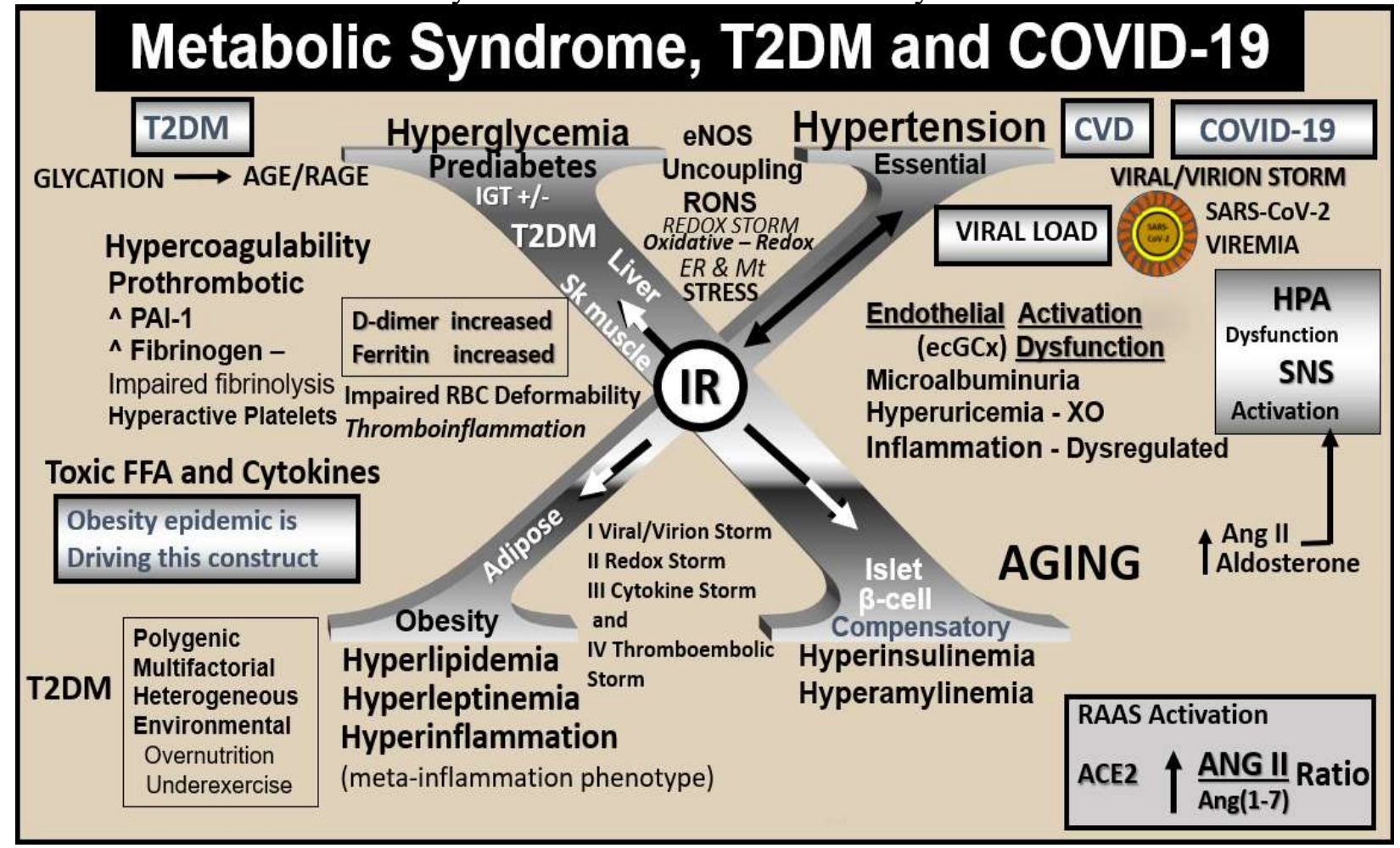

Figure 17. The metabolic Syndrome, type 2 diabetes mellitus (T2DM), COVID-19 and viremia. This illustration portrays the original X of Reaven's syndrome X later renamed the metabolic syndrome by Grundy $[103,104]$. Note the multiple H phenomenon or hyper states associated with each arm of the letter X: hyperlipidemia, hyperinsulinemia, hypertension and hyperglycemia. Further note the central location of insulin resistance (IR) and how it is related to each arm of the letter X. These multiple variables all come together and interact with the associated multiple variables of the SARS-CoV-2 viremia of the COVID-19 pandemic. It is important to note that the obesity epidemic as noted in the left lower part of this illustration may be driving this construct in different parts of the world. $A C E 2=$ angiotensin-converting enzyme 2; $A G E=$ advanced glycation end products; $A N G I I / A n g I I=$ angiotensin II; Ang(1-7) = angiotensin 1-7; CVD = cerebro-cardiovascular disease; D-dimer = a fibrin degradation product and is named after two D fragments of the fibrin protein joined by a crosslink upon fibrinolysis; ecGCx = endothelial glycocalyx; eNOS = endothelial nitric oxide synthase; $E R=$ endoplasmic; FFA free fatty acid; $M t=$ mitochondria; $P A I-1=$ plasminogen activator-1; $R A G E=$ receptor for $A G E ; R B C=$ red blood cell; $R O N S=$ reactive oxygen and nitrogen (nitrosative stress) species; $S N S=$ sympathetic nervous system; $X O=$ xanthine oxidase.

Indeed, this SARS-CoV-2 virus is not through with us at this time and it is anticipated that there will be an increasing number of cases and increasing mortality in the months ahead. While this Christmas and winter season seems to be darkened by the COVID-19 pandemic, it seems like there may be dark times ahead as we enter the annual active influenza winter season. However, we must remain positive in knowing if we can see it, we can study it and advance knowledge through a better understanding of how to better treat and survive this devastating COVID-19 pandemic of 2019-2020. Importantly, the world is now able to see some light since the COVID-19 vaccines are just beginning to rollout globally and our highly vulnerable health care teams are currently 
being inoculated. In the coming new year of 2021, we should all begin to see a light in all this darkness we have endured, while never forgetting the ones we have lost, for they are not lost if we keep them in our memory and our hearts.

Acknowledgments: Author wishes to acknowledge all of the lost souls due to this pandemic along with their supportive families who have lost loved ones in addition to the heroic first responders, nurses, doctors, health care providers and those who make a hospital and administration run efficiently. The author also wishes to thank the University of Missouri, Columbia, Missouri electron microscopy core center over the many years of assisting in the preparation of tissues and guidance.

Author contributions: $\mathrm{MRH}$ is totally responsible for the contents of this review, writing and editing.

Ethics: The tissues provided for the representative electron microscopic images utilized in this manuscript were all approved in advance by the University of Missouri Institutional Animal Care and Use Committee, and animals were cared for in accordance with National Institutes of Health guidelines and by the Institutional Animal Care and Use Committees at the Harry S Truman Memorial Veterans' Hospital and University of Missouri, Columbia, MO, USA and conformed to the Guide for the Care and Use of Laboratory Animals published by the National Institutes of Health (NIH).

Declaration of conflicting interest: The author declares that there is no conflict of interest.

Funding: This manuscript received no grants from any funding agency in the public, commercial, or not-for-profit sectors.

\section{References}

1. Zhu, N.; Zhang, D.; Wang, W.; Li, X.; Yang, B.; Song, J.; Zhao, X.; Huang, B.; Shi, R.L.; Lu, R. et al. A Novel Coronavirus from Patients with Pneumonia in China, 2019. China Novel Coronavirus Investigating and Research Team. N Engl J Med. 2020 Feb 20;382(8):727-733. doi: 10.1056/NEJMoa2001017

2. Zhou, P.; Yang, X.L.; Wang, X.G.; Hu, B.; Zhang, L.; Zhang, W.; Si, H.R.; Zhu, Y.; Li, B.; Huang, CL. et. al. A pneumonia outbreak associated with a new coronavirus of probable bat origin. Nature. 2020 Mar;579(7798):270-273. doi: 10.1038/s41586-020-2012-7

3. The New York Times. Coronavirus in the U.S.: Latest Map and Case Count. Available online: https://www.nytimes.com/interactive/2020/us/coronavirus-us-cases.html (accessed on 23 December, 2020) 
4. World Health Organization; Coronavirus disease (COVID-19). https://www.who.int/docs/default-source/coronaviruse/situation-reports/20201012-weeklyepi-update-9.pdf (last accessed on 23 December, 2020)

5. 2009 H1N1 Pandemic (H1N1pdm09 virus). https:/www.cdc.gov/flu/pandemicresources/2009-h1n1-pandemic.html (last accessed on 23 December, 2020)

6. Hayden, M.R. Endothelial activation and dysfunction in metabolic syndrome, type 2 diabetes and coronavirus disease 2019. J Int Med Res. July 2020; doi: 10.1177/0300060520939746

7. 1918 Pandemic (H1N1 virus). https://www.cdc.gov/flu/pandemic-resources/1918-pandemich1n1.html (last accessed on 23 December, 2020)

8. West, J.B. Comparative physiology of the pulmonary blood-gas barrier: the unique avian solution. Am J Physiol Regul Integr Comp Physiol. 2009 Dec; 297(6): R1625-R1634. doi: 10.1152/ajpregu.00459.2009

9. Makanya, A.; Anagnostopoulou, A.; Djonov V. Development and Remodeling of the Vertebrate Blood-Gas Barrier. BioMed Research Int. 2013; 2013:101597. doi:10.1155/2013/101597

10. Wagner, P.D. The physiological basis of pulmonary gas exchange: implications for clinical interpretation of arterial blood gases. European Respiratory Journal. 2015; 45: 227243; doi: 10.1183/09031936.00039214

11. Subbarao, K.; Mahanty, S. Respiratory Virus Infections: Understanding COVID-19. Immunity. 2020 Jun 16; 52(6): 905-909. doi: 10.1016/j.immuni.2020.05.004

12. Bao, L.; Zhang, C.; Dong, J.; Zhao, L.; Li, Y.; Sun,; J. Oral Microbiome and SARS-CoV-2: Beware of Lung Co-infection. Front Microbiol. 2020; 11: 1840. doi: 10.3389/fmicb.2020.01840

13. Lozach, P.Y. Cell Biology of Viral Infections. Cells. 2020 Nov 7;9(11):2431. doi: 10.3390/cells9112431 
14. Coburn, B.J.; Wagner, B.G; Blower, S. Modeling influenza epidemics and pandemics: insights into the future of swine flu (H1N1). BMC Med. 2009;7:30. doi: 10.1186/1741-70157-30

15. Sanche, S.; Lin, Y.T.; Xu, C.; Romero-Severson, E.; Hengartner, N.; Ke, R. High Contagiousness and Rapid Spread of Severe Acute Respiratory Syndrome Coronavirus 2. Emerg Infect Dis. 2020 Jul;26(7):1470-1477. doi: 10.3201/eid2607.200282

16. Xu, Z.; Shi, L.; Wang, Y.; Zhang, I.; Huang, L.; Zhang, C.; Liu, S.; Zhao, P.; Liu, H.; Zhu, L. et al. Pathological findings of COVID-19 associated with acute respiratory distress syndrome. Lancet Respir Med. 2020 Apr;8(4):420-422. doi: 10.1016/S2213-2600(20)30076$\mathrm{X}$

17. Mason, R.J. Pathogenesis of COVID-19 from a cell biology perspective. European Respiratory Journal. 2020; 55:2000607. doi: 10.1183/13993003.00607-2020

18. Hogan, C.A.; Stevens, B.; Sahoo, M.K.; Huang, CH.; Garamani, N.; Gombar, S.; Yamamoto, F.; Murugesan, K.; Kurzer, J. et al. High frequency of SARS-CoV-2 RNAemia and association with severe disease. medRxiv preprint. 2020 ; doi: https://doi.org/10.1101/2020.04.26.20080101

19. Carsana, L.; Sonzogni, A.; Nasr, A,; Rossi, R.S.: Pellegrinelli, A.; Zerbi, P.; Rech, R.; Colombo, R,; Antinori, S.; Corbellino, M.; et al. Pulmonary post-mortem findings in a series of COVID-19 cases from northern Italy: a two-centre descriptive study. Lancet Infect Dis. 2020 Oct;20(10):1135-1140. doi: 10.1016/S1473-3099(20)30434-5

20. Hayden, M.R. An Immediate and Long-Term Complication of COVID-19 May Be Type 2 Diabetes Mellitus: The Central Role of $\beta$-Cell Dysfunction, Apoptosis and Exploration of Possible Mechanisms. Cells 2020, 9, 2475. https://doi.org/10.3390/cells9112475

21. Tan C, Li S, Liang Y, Chen M, Liu J. SARS-CoV-2 viremia may predict rapid deterioration of COVID-19 patients. Braz J Infect Dis. 2020;S1413-8670(20)30138-0. doi: 10.1016/j.bjid.2020.08.010 10.1016/j.bjid.2020.08.010

22. Di Cristanziano, V.; Meyer-Schmicker, C.; Eberhardt, K.A.; E=Rybniker, I.; Heger, E.; Knops, E.; Hallek, M.; Klein, F.; Holtick, U.; Jung, N. Detection of SARS-CoV-2 viremia before onset of COVID-19 symptoms in an allo-transplanted patient with acute leukemia. Bone Marrow Transplant. (2020). https://doi.org/10.1038/s41409-020-01059-y

23. Bermejo-Martin, J.F.; González-Rivera, M.; Almansa, R.; et al. Viral RNA load in plasma is associated with critical illness and a dysregulated host response in COVID-19. medRxiv preprint. October 27 2020. doi: https://doi.org/10.1101/2020.08.25.20154252

24. Fajnzylber, J.; Regan, J.; Coxen,; Corry, H.; 1,6, Wong, C.; Rosenthal, A.; Worrall, D.; Giguel, F.; Piechocka-Trocha, A.; Atyeo, C. SARS-CoV-2 viral load is associated with 
increased disease severity and mortality. Nat Commun 11, 5493 (2020). https://doi.org/10.1038/s41467-020-

25. Chen, X.; Zhao, B.; Qu, Y.; Chen, Y.; Xiong, J.; Feng, Y.; Men, D.; Huang, Q.; Liu, Y.; Yang, B. Detectable serum SARS-CoV-2 viral load (RNAaemia) is closely correlated with drastically elevated interleukin 6 (IL-6) level in critically ill COVID-19 patients. Clin Infect Dis. 2020; 71(8):1937-1942. doi:10.1093/cid/ciaa449

26. Gallo, O.; Locatello, L.G.; Mazzoni, A,; Novelli, L.; Annunziato, F. The central role of the nasal microenvironment in the transmission, modulation, and clinical progression of SARSCoV-2 infection. Mucosal Immunol. 2020 Nov 26: 1-12. doi: 10.1038/s41385-020-00359-2

27. Chowdhury, M.A.; Hossain, N.; Kashem, M.A.; Shahid, A.; Alam, A. Immune response in COVID-19: A review. Journal of Infection and Public Health. 2020 Nov;13(11):1619-1629. https://doi.org/10.1016/j.jiph.2020.07.001

28. Liu, L.; Wei, Q.; Nishiura, K.J.; Wang, H.; Midkiff,C.; Alvarez, X.; Qin, C.; Lackner, A.; Chen, Z. Spatiotemporal interplay of severe acute respiratory syndrome coronavirus and respiratory mucosal cells drives viral dissemination in rhesus macaques. Mucosal Immunol. 2015; 9, 1089-1101 (2016). https://doi.org/10.1038/mi.2015.127

29. Boulenger, N.; Samardo, T.; Abkari, M.; Abkari, S.; Celeries, C.; Famous. P.; Luscan, R.; Mansbach, A. L.; Moreddu, E.; Pondaven S. et al. COVID-19 and ENT Pediatric otolaryngology during the COVID-19 pandemic. French Association of Pediatric Otorhinolaryngology (AFOP); French Society of Otorhinolaryngology; Head, Neck Surgery (SFORL). Eur Ann Otorhinolaryngol Head Neck Dis. 2020 May;137(3):177-181. doi: 10.1016/j.anorl.2020.04.010

30. Pondaven-Letourmy, S.; Alvin, F.; Boumghit, Y.; Simon, F. How to perform a nasopharyngeal swab in adults and Coronavirus. Available online: https://www.cdc.gov/coronavirus/2019-ncov/symptoms-testing/symptoms.html (accessed on 23 December 2020)

31. Zacharioudakis, I.M.; Prasad. P.J.; Zervou, F.N.; Basu, A.; Inglima, K.; Weisenberg, S.A.; Aguero-Rosenfeld, M.E. Association of SARS-CoV-2 Genomic Load with COVID-19 Patient Outcomes. Ann Am Thorac Soc. 2020 Oct 29. doi: 10.1513/AnnalsATS.202008-931RL 
32. Centers for Disease Control and Prevention. Symptoms of Coronavirus. Available online: https://www.cdc.gov/coronavirus/2019-ncov/symptoms-testing/symptoms.html (accessed on 23 December 2020)

33. World Health Organization. Coronavirus Symptoms. Available online: https://www.who.int/health-topics/coronavirus\#tab=tab_3 (accessed on 23 December 2020)

34. Berradja, N.; Edjlali, M.; Carlier, R.; Hans, S. MRI of Patients Infected With COVID-19 Revealed Cervical Lymphadenopathy. Ear Nose Throat J. 2020 Jul 23:145561320940117. doi: $10.1177 / 0145561320940117$.

35. Lechien, J.R.; Estomba, C.M.; De Siati, D.R.; Horoi, M.; Le Bon, S.D.; Rodriguez, A.; Dequanter, D.; Blecic, S.; El Afia, F.; Distinguin, L.; et al. Olfactory and gustatory dysfunctions as a clinical presentation of mild-to moderate forms of the coronavirus disease (COVID-19): a multicenter European study [published online ahead of print April 6, 2020]. Eur Arch OtoRhinol. 2020; doi:10.1007/s00405-020-05965-1

36. Lechien, J.R.; Chiesa-Estomba, C.M.; Place, S.; Van Laethem, Y.; Cabaraux, P.; Mat, Q.; Huet, K.; Plzak, J.; Horoi, M.; Hans, S.; et al. Clinical and epidemiological characteristics of 1,420 European patients with mild-to-moderate coronavirus disease 2019. J Intern Med. 2020; doi:10.1111/joim. 13089

37. Kaneko, N.; Hsiao-Hsuan Kuo, H.H.; Boucau, J.; Farmer, J.R.; Allard-Chamard, H.; Mahajan, V.S.; Piechocka-Trocha, A.; Lefteri, K.; Osborn, M.; Bals, J. Loss of Bcl-6Expressing T Follicular Helper Cells and Germinal Centers in COVID-19. Cell. 2020 Oct 1; 183(1): 143-157.e13. doi: 10.1016/j.cell.2020.08.025

38. Xu, X.; Chang, X.N.; Pan, H.; Su, H.; Hyang, B.; Yang, M.; Luo, D. J.; Weng, M.X.; Ma, L.; Nie X. Pathological changes of the spleen in ten patients with coronavirus disease 2019(COVID-19) by postmortem needle autopsy. Chinese Journal of Pathology, 26 Apr 2020, 49(6):576-582 doi: 10.3760/cma.j.cn112151-20200401-00278

39. Petrova, T. B.; Koh, G.Y. Biological functions of lymphatic vessels. Science. 2020 Jul 10;369(6500): eaax4063. doi: 10.1126/science.aax4063 
40. Hu, B.; Guo, H.; Zhou, P.; Shi, ZL. Characteristics of SARS-CoV-2 and COVID-19. Nat Rev Microbiol. 2020 Oct 6:1-14. doi: 10.1038/s41579-020-00459-7

41. Bradley, B.T.; Johnston, R.; Chaudhry, I.; MD Susan L Fink, MD Prof Haodong Xu, MD Behzad Najafian, MD Prof Gail Deutsch, MD J Matthew Lacy, MD Timothy Williams, MD et al. Histopathology and ultrastructural findings of fatal COVID-19 infections in Washington State: a case series. Lancet. 2020396 (10247), P320-332, AUGUST 01, 2020 https://doi.org/10.1016/S0140-6736(20)31305-2

42. Centers for Disease Control and Prevention. 2020. Interim guidelines for collecting, handling, and testing clinical specimens for COVID-19. Available online:

https://www.cdc.gov/coronavirus/2019-ncov/lab/guidelines-clinical-specimens.html (accessed on 10 December 2020)

43. Hanson KE, Barker AP, Hillyard D.R.; Gilmore, N.; Barrett, J.W.; R. R.; Orlandi, R.R; Shakir, S.M. Self-Collected Anterior Nasal and Saliva Specimens versus Health Care WorkerCollected Nasopharyngeal Swabs for the Molecular Detection of SARS-CoV-2. J Clin Microbiol. 2020 Oct 21;58(11):e01824-20. doi: 10.1128/JCM.01824-20

44. Inagami, T.; Naruse, M.; Hoover, R. Endothelium as an endocrine organ. Annu. Rev. Physiol. 1995, 57, 171-189. doi: 10.1146/annurev.ph.57.030195.001131

45. Flammer, A.J.; Anderson, T.; Celermajer, D.S.; Creager, M.A.; Deanfield, J.; Ganz, P.; Hamburg, N.M.; Lüscher, T.F.; Shechter, M.; Taddei, S.; et al. The assessment of endothelial function: from research into clinical practice. Circulation. 2012 Aug 7;126(6):753-67. doi: 10.1161/CIRCULATIONAHA.112.093245

46. Libby, P.; Lüscher, T. COVID-19 is, in the end, an endothelial disease. Eur Heart J. 2020 Aug 21; 41(32): 3038-3044. 2020 Sep 3. doi: 10.1093/eurheartj/ehaa623

47. Siddiqi, K.M.; Libby, P.; Ridker, P.M. COVID-19 - A vascular disease. Trends Cardiovasc Med. 2020 Oct14:S1050-1738(20)30128-6. doi: 10.1016/j.tcm.2020.10.005

48. Willms-Kretschmer, K.; Flax, M.H.; Cotran, R.S. The fine structure of the vascular response in hapten-specific delayed hypersensitivity and contact dermatitis. Lab Invest. 1967 Sep; 17(3):334-49. 
49. Pober, J.S. Warner-Lambert/Parke-Davis award lecture. Cytokine-mediated activation of vascular endothelium. Physiology and pathology. Am J Pathol. 1988 Dec; 133(3):426-33.

50. Pober, J.S.; Sessa, W.C. Evolving functions of endothelial cells in inflammation. Nat Rev Immunol, 2917; 7, 803-815, https://doi.org/10.1038/nri2171

51. Hunt, B.J.; Jurd, K.M. Endothelial cell activation A central pathophysiological process. BMJ. 1998 May 2; 316(7141): 1328-1329. doi: 10.1136/bmj.316.7141.1328

52. Hayden, M.R.; Habibi, J.; Joginpally, T.; Karuparthi, P.R.; Sowers, J.R. Ultrastructure Study of Transgenic Ren2 Rat Aorta - Part 1: Endothelium and Intima. Cardiorenal Med. 2012 Feb;2(1):66-82. doi: 10.1159/000335565

53. Giannotta, M.; Trani, M.; Dejana, E. VE-cadherin and endothelial adherens junctions: active guardians of vascular integrity. Dev Cell. 2013 Sep 16;26(5):441-54. doi:

10.1016/j.devcel.2013.08.020

54. Hayden, M.R.; Sowers, J.R.; Suresh C Tyagi, S. C. The central role of vascular extracellular matrix and basement membrane remodeling in metabolic syndrome and type 2 diabetes: the matrix preloaded. Cardiovasc Diabetol. 2005 Jun 28;4:9. doi: 10.1186/1475-2840-4-9

55. Reitsma, S.; Slaaf, D.W.; Vink, H.; van Zandvoort, M.A.; oude Egbrink, M.G. The endothelial glycocalyx: composition, functions, and visualization. Pflugers Arch. 2007 Jun;454(3):345-59. doi: 10.1007/s00424-007-0212-8

56. Luft, J.H. Fine structures of capillary and endocapillary layer as revealed by ruthenium red. Fed Proc. 1966; 25:1773-1783. PMID: 5927412

57. Pries, A.R.; Secomb. T.W.; Gaehtgens, P. The endothelial surface layer. Pflugers Arch. 2000 Sep; 440(5):653-666. doi: 10.1007/s004240000307

58. Inagawa, R,; Okada, H.; Takemura, G.; Kodai Suzuki, K.; Chihiro Takada, C.; Hirohisa Yano, H.; Ando, Y.; Usui, T.; Hotta,Y.; Miyazaki, N.; et. al. Ultrastructural Alteration of Pulmonary Capillary Endothelial Glycocalyx During Endotoxemia. Chest. 2018 Aug;154(2):317-325. doi: 10.1016/j.chest.2018.03.003 
59. Ando Y., Okada H., Takemura G., Suzuki K., Takada C., Tomita H., Zaikokuji R., Hotta Y., Miyazaki N., Yano H., et al. Brain-Specific Ultrastructure of Capillary Endothelial Glycocalyx and Its Possible Contribution for Blood Brain Barrier. Sci. Rep. 2018;8:17523. doi: 10.1038/s41598-018-35976-2

60. Hayden, M.R. Type 2 Diabetes Mellitus Increases The Risk of Late-Onset Alzheimer's Disease: Ultrastructural Remodeling of the Neurovascular Unit and Diabetic Gliopathy. Brain Sci. 2019 Sep 29;9(10):262. doi: 10.3390/brainsci9100262

61. Yamaoka-Tojo, M. Endothelial glycocalyx damage as a systemic inflammatory microvascular endotheliopathy in COVID-19. Biomed J. 2020 Oct; 43(5): 399-413. doi: 10.1016/j.bj.2020.08.007

62. Okada, H.; Yoshida, S.; Hara, A.; Ogura, S.; Tomita, H. Vascular endothelial injury exacerbates coronavirus disease 2019: The role of endothelial glycocalyx protection. Microcirculation. 13 August 2020 https://doi.org/10.1111/micc.12654

63. Wadman, M.; Couzin-Frankel, J.; Kaiser, J.; Matacic. C. A rampage through the body. Science. 2020 Apr 24;368(6489):356-360. doi: 10.1126/science.368.6489.356

64. Pretorius, E. The adaptability of red blood cells. Cardiovascular Diabetology. 2013, 12:63. doi: $10.1186 / 1475-2840-12-63$

65. Archer, N.; Shmukler, B.; Andolfo, I.; Vandorpe, D.; Gnanasambandam, R.; Higgins, J.; et al. Hereditary xerocytosis revisited. Am. J. Hematol.2014;89:1142-1146. doi:10.1002/ajh.23799

66. Mohandas, N.; Gallagher, P.G. Red cell membrane: past, present, and future. Blood. 2008 Nov 15;112(10):3939-48. doi: 10.1182/blood-2008-07-161166

67. Wang, K,; Chen, W.; Zhom, Y.S. SARS-CoV-2 invades host cells via a novel route: CD147spike protein. bioRxiv preprint. March 14, 2020; doi: https://doi.org/10.1101/2020.03.14.988345 
68. Ulrich, H.; Pillat, MM.; CD147 as a Target for COVID-19 Treatment: Suggested Effects of Azithromycin and Stem Cell Engagement. Stem Cell Rev Rep. 2020 Apr 20: 1-7.

doi: $10.1007 / \mathrm{s} 12015-020-09976-7$

69. Shilts, J.; Wright, GJ. No evidence for basigin/CD147. bioRxiv preprint. July 26, 2020; doi: https://doi.org/10.1101/2020.07.25.221036

70. Thomas, T.; Stefanoni, D.; Dzieciatkowska, M.; Issaian, A.; Nemkov, T.; Hill, R.C.; Francis, R.O.; Hudson, K.E.; Buehler, P.W.; Zimring, J.C.; et al. Evidence for structural protein damage and membrane lipid remodeling in red blood cells from COVID-19 patients. medRxiv preprint. 2020; doi: https://doi.org/10.1101/2020.06.29.20142703

71. Yoshida, T.; Prudent, M.; D’Alessandro, A. Red blood cell storage lesion: causes and potential clinical consequences. Blood Transfus. 2019;17(1):27-52. doi:10.2450/2019.021718

72. Buys, A.V.; Van Rooy, M.J.; Soma, P.; Van Papendorp, D.; Lipinski, B.; Pretorius, E. Changes in red blood cell membrane structure in type 2 diabetes: a scanning electron and atomic force microscopy study. Cardiovascular Diabetology 2013, 12:25. doi: http://www.cardiab.com/content/12/1/25

73. Aydin, H.; Al-Khooly, D.; Lee, JE. Influence of hydrophobic and electrostatic residues on SARS-coronavirus S2 protein stability: Insights into mechanisms of general viral fusion and inhibitor design. Protein Sci. 2014 May; 23(5): 603-617. doi: 10.1002/pro.2442

74. Pretorius, E. The adaptability of red blood cells. Cardiovasc Diabetol. 12, 63 (2013). https://doi.org/10.1186/1475-2840-12-63

75. Cho, Y.I.; Mooney, M.P.; Cho, DJ. Hemorheological Disorders in Diabetes Mellitus. $J$ Diabetes Sci Technol. 2008 Nov; 2(6): 1130-1138. 10.1177/193229680800200622

76. Danese, E; Lippi, G.; Montagnana, M. Red blood cell distribution width and cardiovascular diseases. J Thorac Dis. 2015;7(10):E402-E411. doi: 10.3978/j.issn.2072-1439.2015.10.04

77. Cavezzi, A.; Troiani, E.; Corrao, S. COVID-19: hemoglobin, iron, and hypoxia beyond inflammation. A narrative review. Clin Pract. 2020 May 19; 10(2): 1271. doi: $10.4081 /$ cp.2020.1271 
78. Pretini. V.; Koenen, M.H.; Kaestner, L.; Fens, M.H.A.A.M.; Scheffelers, R.M.; Bartels, M.; Van Wijk, R. Red Blood Cells: Chasing Interactions. Front. Physiol. 2019; 10: 945. doi: https://doi.org/10.3389/fphys.2019.00945

79. Gu, S.X.; Tyagi, T.; Jain, K.; Gu, V.W.; Lee, S. H.; Hwa, I. M.; Kwan, J.M.; Krause, D.S.; Lee, A.I.; Halene, S. et al. Thrombocytopathy and endotheliopathy: crucial contributors to COVID-19 thromboinflammation. Nat Rev Cardiol (2020). https://doi.org/10.1038/s41569020-00469-1

80. Thon, J.N.; Italiano, J.E. PLATELET FORMATION. Semin Hematol. 2010 Jul; 47(3): 220226. doi: $10.1053 /$ j.seminhematol.2010.03.005

81. Xu, P.; Zhou, Q.; Xu,; J. Mechanism of thrombocytopenia in COVID-19 patients. Ann Hematol. 2020; 99(6): 1205-1208. doi: 10.1007/s00277-020-04019-0

82. Hottz, E.D.; Azevedo-Quintanilha, I.G.; Palhinha, L.; Teixeira, L.; Barreto, E.A.; Pão, C.R.R.; Righy, C.; Franco, S.; Souza, T.M.L.; Pedro Kurtz, P.; et al. Platelet activation and platelet-monocyte aggregate formation trigger tissue factor expression in patients with severe COVID-19. Blood. 2020 Sep 10;136(11):1330-1341. doi: 10.1182/blood.2020007252

83. Manfred Kopf, M.; Schneider, C.; Nobs, S.P. The development and function of lung-resident macrophages and dendritic cells. Nat Immunol. 2015 Jan;16(1):36-44. doi: 10.1038/ni.3052

84. Merad, M.; Martin, J.C. Pathological inflammation in patients with COVID-19: a key role for monocytes and macrophages. Nat Rev Immunol. 2020 Jun;20(6):355-362. doi: 10.1038/s41577-020-0331-4

85. Martinez, F.O.; Combes, T.W.; Orsenigo, F.; Gordon, S. Monocyte activation in systemic Covid-19 infection: Assay and rationale. EBioMedicine. 2020; Sep;59:102964. doi: 10.1016/j.ebiom.2020.102964

86. Gómez-Rial, J.; Rivero-Calle, I.; Salas, A.; Martinón-Torres, F. Role of Monocytes/Macrophages in Covid-19 Pathogenesis: Implications for Therapy. Infect Drug Resist. 2020 Jul 22;13:2485-2493. doi: 10.2147/IDR.S258639 
87. Kumar, K.P.; Alyce, J.; Nicholls, A.J.; Wong, C.H.Y. Partners in crime: neutrophils and monocytes/macrophages in inflammation and disease. Cell Tissue Res. 2018; 371(3): 551565. doi: 10.1007/s00441-017-2753-2

88. Tomar, B.; Anders, J.H.; Desai, J.; Mulay, S.R. Neutrophils and Neutrophil Extracellular Traps Drive Necroinflammation in COVID-19. Cells. 2020 June; Cells 2020, 9, 1383; doi:10.3390/cells9061383

89. Arcanjo, A.; Logullo, J.; Menezes, C.C.B.; de Souza Carvalho Giangiarulo, T.C.; Dos Reis, M.C.; de Castro, G. M. M.; da Silva Fontes, Y.; Todeschini, A.R.; Freire-de-Lima, L.

Decoté-Ricardo, D.; et al. The emerging role of neutrophil extracellular traps in severe acute respiratory syndrome coronavirus 2 (COVID-19). Sci Rep. 2020 Nov 12;10(1):19630. doi: $10.1038 / \mathrm{s} 41598-020-76781-0$

90. Lecube, A.; Pachón, G.; Petriz, J.; Hernández, C.; Simó, R. Phagocytic Activity Is Impaired in Type 2 Diabetes Mellitus and Increases after Metabolic Improvement. PLoS One. 2011; 6(8): e23366.doi: 10.1371/journal.pone.0023366

91. Zuo, Y.; Zuo, M.; Yalavarthi, S.; Gockman, K.; Madison, J.A.; Shi, H.; Woodard, W.; S.P.; Lugogo, N.L.; Knight J.S. Neutrophil extracellular traps and thrombosis in COVID-19. J Thromb Thrombolysis. 2020 Nov 5: 1-8. doi: 10.1007/s11239-020-02324-Z

92. Rao, V.U.S.; Arakeri, G.; Subash, A.; Rao,J.; Jadhav, S.; Sayeed, M. S.; Rao, G.; Brennan, P.A. COVID-19: Loss of bridging between innate and adaptive immunity? Med Hypotheses. 2020 Nov; 144: 109861 doi: 10.1016/j.mehy.2020.109861

93. Alberts, B.; Johnson, A.; Lewis, J.; Raff, M.; Roberts, K.; Walter, P. Molecular Biology of the Cell, 4th edition. Garland Science; New York: 2002. Lymphocytes and the Cellular Basis of Adaptive Immunity. ISBN-10: 0-8153-3218-1ISBN-10: 0-8153-4072-9

94. Yang, L.; Liu, S.; Liu, I.; Zhang, Z.; Wan, X.; Haung, B.; Chen, Y.; Zhang, Y. COVID-19: immunopathogenesis and Immunotherapeutics. Signal Transduct Target Ther. $2020 \mathrm{Jul}$ 25;5(1):128. doi: 10.1038/s41392-020-00243-2

95. Li, T.; Qiu, Z.; Zhang, L.; Han, Y.; He, W.; Liu, Z.; Ma. X.; Fan, H.; Lu, W.; Xie, J.; et al. J Infect Dis. 2004 Feb 15;189(4):648-51. doi: 10.1086/381535

96. Huang, C.; Wang, Y.; Li, X.; Ren, L.; Zhao, I.; Hu, Y.; Zhang, L.; Fan, G.; Xu, J.; Gu, X.; et al. Clinical features of patients infected with 2019 novel coronavirus in Wuhan, China. Lancet. 2020; 395:497-506. doi:10.1016/S0140-6736(20)30183-5 
97. Schub, D.; Klemis, V.; Schneitler, S.; Mihm, J.; Lepper, P.M.; Wilkins, H.; Bals, R.; Eichler, H.; Gaertner, B.C.; Becker, S.L. et al. High levels of SARS-CoV-2 specific T-cells with restricted functionality in patients with severe course of COVID-19. medRxiv preprint, 2020; doi: https://doi.org/10.1101/2020.07.08.20148718

98. Haung I.; Pranata R. Lymphopenia in severe coronavirus disease-2019 (COVID-19): systematic review and meta-analysis. $j$ intensive care 8, 36 (2020). doi: https://doi.org/10.1186/s40560-020-00453-4

99. Li, H.; Luo, M.; Liu, J.; Wu, L.; Lin, X.; Li, R.; Wang, Z.; Zhong, H.; Zheng, W.; et al. Lymphopenia predicted illness severity and recovery in patients with COVID-19: A singlecenter, retrospective study. PLoS One. 2020 Nov18;15(11):e0241659. doi: 10.1371/journal.pone.0241659

100. Fenner, F.; Bachmann, P.A.: Gibbs, P.J.; Murphy, F.A.; Studdert, M.J.; White D.O. Pathogenesis: Infection and the Spread of Viruses in the Body. Veterinary Virology. 1987: 133-152. 2014 Jun 27. doi: 10.1016/B978-0-12-253055-5.50011-6

101. Belanger, M.J.; Hill, M.A.; Angelidi, A.M.; Dalamaga, M.; Sowers, J. R.; Mantzoros, C.S. Covid-19 and Disparities in Nutrition and Obesity. N Engl J Med. 2020; 383(11):e69. doi: 10.1056/NEJMp2021264

102. Gupta A.; Madhavan, M.V.; Sehgal, K.; Nair, N.; Mahajan, S.; Sehrawat, T.S.; Bikdeli, B.; Shluwalia, N.; Ausiello, J.C.; Wan, E.Y. et al. Extrapulmonary manifestations of COVID-19. Nat Med. 2020; 26, https://doi.org/10.1038/s41591-020-0968-3

103. Reaven, G.M. Banting lecture 1988. Role of insulin resistance in human disease. Diabetes. 1988 Dec;37(12):1595-607. doi: 10.2337/diab.37.12.1595

104. Scott M. Grundy, S.M. Metabolic Syndrome: A Multiplex Cardiovascular Risk Factor. $J$ Clin Endocrinol Metab. 2007 Feb;92(2):399-404. doi: 10.1210/jc.2006-051 